\title{
Deletion of a Csfir enhancer selectively impacts CSF1R expression and development of tissue macrophage populations
}

\author{
Rocío Rojo (1) 1,2, Anna Raper (10 1, Derya D. Ozdemir', Lucas Lefevre (1) 1, Kathleen Grabert (10) 1,3, \\ Evi Wollscheid-Lengeling1, Barry Bradford (10 1, Melanie Caruso (1) 1, Iveta Gazova (1) 1, Alejandra Sánchez ${ }^{1}$ \\ Zofia M. Lisowski (10 1, Joana Alves (10 1, Irene Molina-Gonzalez (10) 4, Hayk Davtyan (1) 5, Rebecca J. Lodge ${ }^{6}$, \\ James D. Glover ${ }^{1}$, Robert Wallace ${ }^{7}$, David A.D. Munro ${ }^{8}$, Eyal David ${ }^{9}$, Ido Amit ${ }^{9}{ }^{9}$, Véronique E. Miron ${ }^{4}$, \\ Josef Priller ${ }^{8}$, Stephen J. Jenkins (10 ${ }^{6}$, Giles E. Hardingham ${ }^{8,10}$, Mathew Blurton-Jones (1) ${ }^{5}$, Neil A. Mabbott (1) ${ }^{1}$, \\ Kim M. Summers (11) ${ }^{11}$, Peter Hohenstein ${ }^{1,12}$, David A. Hume ${ }^{11,14} \&$ Clare Pridans (1D) 6,13,14
}

The proliferation, differentiation and survival of mononuclear phagocytes depend on signals from the receptor for macrophage colony-stimulating factor, CSF1R. The mammalian Csf1r locus contains a highly conserved super-enhancer, the fms-intronic regulatory element (FIRE). Here we show that genomic deletion of FIRE in mice selectively impacts CSF1R expression and tissue macrophage development in specific tissues. Deletion of FIRE ablates macrophage development from murine embryonic stem cells. Csf1r $\mathrm{FIRE} / \Delta \mathrm{FIRE}$ mice lack macrophages in the embryo, brain microglia and resident macrophages in the skin, kidney, heart and peritoneum. The homeostasis of other macrophage populations and monocytes is unaffected, but monocytes and their progenitors in bone marrow lack surface CSF1R. Finally, Csf1r $\Delta$ FIRE/ $\triangle F I R E$ mice are healthy and fertile without the growth, neurological or developmental abnormalities reported in $\mathrm{Csf1r}^{-/}$- rodents. Csf1r $\mathrm{AFIRE/ \Delta FIRE}$ mice thus provide a model to explore the homeostatic, physiological and immunological functions of tissue-specific macrophage populations in adult animals.

\footnotetext{
${ }^{1}$ The Roslin Institute \& Royal (Dick) School of Veterinary Studies, University of Edinburgh, Easter Bush, Midlothian EH25 9RG, UK. ${ }^{2}$ Tecnologico de Monterrey, Escuela de Medicina y Ciencias de la Salud, Av. Ignacio Morones Prieto 3000 Pte, Col. Los Doctores, C.P. 64710 Monterrey, N.L., Mexico. ${ }^{3}$ Department of Environmental Medicine, Toxicology Unit, Karolinska Institutet, Box 210SE-171 77 Stockholm, Sweden. ${ }^{4}$ The MRC University of Edinburgh Centre for Reproductive Health, The Queen's Medical Research Institute, Edinburgh BioQuarter, 47 Little France Crescent, Edinburgh EH16 4TJ, UK. ${ }^{5}$ Department of Neurobiology and Behavior, University of California Irvine, 3014 Gross Hall 845 Health Sciences Rd, Irvine, CA 92697-1705, USA. ${ }^{6}$ University of Edinburgh Centre for Inflammation Research, The Queen's Medical Research Institute, Edinburgh BioQuarter, 47 Little France Crescent, Edinburgh EH16 4TJ, UK. ${ }^{7}$ The Department of Orthopedic Surgery, University of Edinburgh, Chancellor's Building, Edinburgh BioQuarter, 49 Little France Crescent, Edinburgh EH16 4SB, UK. 8 UK Dementia Research Institute, The University of Edinburgh, Chancellor's Building, Edinburgh BioQuarter, 49 Little France Crescent, Edinburgh EH16 4SB, UK. ${ }^{9}$ Department of Immunology, Weizmann Institute of Science, 234 Herzl St., Rehovot 7610001 , Israel. ${ }^{10}$ Centre for Discovery Brain Sciences, University of Edinburgh, Hugh Robson Building, 15 George Square, Edinburgh EH8 9XD, UK. ${ }^{11}$ Mater Research Institute-University of Queensland, Translational Research Institute, Woolloongabba, QLD 4102, Australia. ${ }^{12}$ Leiden University Medical Center, P.O. Box 96002300 RC Leiden, The Netherlands. ${ }^{13}$ Simons Initiative for the Developing Brain, Centre for Discovery Brain Sciences, University of Edinburgh, Hugh Robson Building, George Square, Edinburgh EH8 9XD, UK. ${ }^{14}$ These authors jointly supervised this work: David A. Hume, Clare Pridans. Correspondence and requests for materials should be addressed to D.A.H. (email: David.Hume@uq.edu.au) or to C.P. (email: clare.pridans@ed.ac.uk)
} 
T he proliferation, differentiation, and survival of vertebrate macrophages is controlled by signals from the macrophage colony-stimulating factor (CSF1) receptor (CSF1R), encoded by the c-fms protooncogene ${ }^{1}$, now known as Csfir. The function of the receptor and its two ligands, CSF1 and interleukin 34 (IL34), in macrophage differentiation is conserved in all amniotes (i.e. reptiles, birds, and humans) ${ }^{2,3}$. Deletion of the Csfir locus in the mouse or rat germ line produces a global deficiency in most tissue macrophage populations ${ }^{4,5}$. Mutant animals are osteopetrotic (associated with loss of osteoclasts) and exhibit severe postnatal growth retardation and multiple pleiotropic impacts on development. Tissue macrophages remain dependent upon CSF1R in adult mice and can be depleted by treatment with an inhibitory anti-CSF1R antibody ${ }^{6}$ or treatment with orally available inhibitors of CSF1R kinase activity ${ }^{7}$. In humans, dominant-negative mutations in the tyrosine kinase domain are associated with an adult-onset neurodegenerative disease $^{8}$.

The expression of Csf1r mRNA is restricted to myeloid cells. During embryonic development, Csf $1 r$ mRNA is expressed in the earliest macrophages identifiable in the yolk sac and transcription of this gene in pluripotent bone marrow (BM) progenitors is the hallmark of commitment to the monocyte-macrophage lineage. Accordingly, the molecular basis for myeloid-restricted transcription regulation of Csfir has been studied in considerable detail (reviewed in ref. ${ }^{9}$ ). The second intron, downstream of the first coding exon, contains a conserved 337bp sequence known as the $f m s$-intronic regulatory element (FIRE), identified as a superenhancer in genome-wide analysis of chromatin in mouse macrophages. The mouse FIRE sequence contains binding sites for numerous macrophage-expressed transcription factors ${ }^{9}$. The combination of a $3.5 \mathrm{~kb}$ Csf1r promoter and intron 2, containing FIRE, is sufficient to direct reproducible transgenic reporter gene expression in progenitors, monocytes, granulocytes, classical dendritic cells, and tissue macrophages that also express Csfir mRNA $^{10,11}$. Removal of FIRE from the reporter construct abolishes expression ${ }^{10}$. The same Csfir construct was used to drive conditional Cre-recombinase in lineage-trace studies that dissected the role of the yolk sac in macrophage development ${ }^{12}$. In mammals, the FIRE sequence is more highly conserved than any of the exons of the Csflr gene ${ }^{13}$. A FIRE sequence is present in the same relative intronic location of the Csf1r locus in all reptile and bird species, and a core element required for enhancer activity ${ }^{14}$ is perfectly conserved ${ }^{13}$. The chicken CSF1R promoter and FIRE sequences are also sufficient to direct macrophage-specific expression in chicken transgenic lines and to highlight the appearance of the earliest phagocyte populations in the yolk sac ${ }^{15}$.

There have been relatively few studies of the function of macrophage-specific enhancers in their genomic context. A highly conserved upstream regulatory element that is present in the Spil locus (which encodes the macrophage-specific transcription factor PU.1) is required for regulated expression in myeloid cells. Deletion of this element in the germline produces a failure of myeloid differentiation and development of acute myeloid leukemia ${ }^{16}$. Deletion of a super-enhancer within the locus of the orphan nuclear receptor transcription factor (Nr4a1) reveals its functional specificity, as only the LY6C ${ }^{\mathrm{lo}}$ monocytes are lost, whilst the sensitivity of other monocyte and macrophage subsets to pro-inflammatory stimuli is preserved ${ }^{17}$.

We aimed to determine the function of FIRE by deleting it from the mouse germ line. If FIRE is essential for Csflr transcription, genomic deletion should partly or completely phenocopy the loss of tissue macrophages observed in Csflr $1 r^{-1-}$ mice. Here we report that deletion of FIRE has an organ-specific effect on Csfir expression and macrophage differentiation. Despite the lack of brain microglia and resident macrophages in skin, heart, kidney, and peritoneum, Csf1 $r^{\Delta \mathrm{FIRE} / \Delta \mathrm{FIRE}}$ mice are healthy and fertile. Aside from highlighting the likely function of other regulatory elements in the Csfir locus, these mice provide a model to explore tissue-specific macrophage transcriptional regulation and function.

\section{Results}

In vitro validation of CRISPRs targeting the FIRE sequence. Guide RNAs (gRNAs) designed to delete FIRE (Fig. 1a) were first validated in the RAW 264.7 macrophage cell line and in E14 mouse embryonic stem cells (ESC). The macrophage cell line expresses Csfir mRNA and was used in all of the transfection studies that previously led to the characterization of the role of FIRE $^{14}$. Both cell types were co-transfected with pairs of Cas9-2AEGFP constructs each expressing single gRNAs (US1+DS1 or DS2), and pools of $\mathrm{EGFP}^{+}$cells were screened via PCR to detect the deletion of FIRE mediated by the CRISPR/Cas9 system. Both pairs of gRNAs produced deletions in E14 ESC and RAW 264.7 cells (Supplementary Fig. 1a). The deletion of FIRE and the absence of mismatches in the remaining Csflr sequence in E14 ESC were confirmed by Sanger sequencing (Supplementary Fig. 1b). In RAW 264.7 macrophage cells, individual Csf1 $r^{\Delta \mathrm{FIRE} / \Delta \mathrm{FIRE}}$ clones carried slightly different deletions of the FIRE sequence (Supplementary Fig. 1c). RAW 264.7 cells are not CSF1R-dependent and most of the cellular CSF1R protein is retained in the Golgi apparatus. We therefore analyzed cell-associated CSF1R by flow cytometry in permeabilized cells. The deletion of FIRE completely ablates detectable intracellular CSF1R (Fig. 1b). There is no impact of the FIRE mutation on cell morphology (Fig. 1c) or phagocytic capacity of cells (Fig. 1d). Neither the frequency nor the median fluorescence intensity (MFI) of the macrophage surface marker F4/80 distinguishes between $C s f 1 r^{+/+}$and $C s f 1 r^{\triangle F I R E} / \Delta$ FIRE clones (Fig. 1e). We conclude that FIRE is required specifically for the expression of CSF1R in the RAW 264.7 macrophage cell line.

E14 mouse ESC can be cultured in the absence of feeders and form embryoid bodies $(\mathrm{EB})^{18}$ with the potential to generate cells belonging to all the primary embryonic germ layers (i.e. endoderm, mesoderm, and ectoderm). Macrophage differentiation from EB was induced by supplementing culture medium with murine IL3 (mIL3) and recombinant human CSF1 (rhCSF1). Csf1 $r^{\Delta \mathrm{FIRE} / \Delta \mathrm{FIRE}}$ mouse ESC generated using the gRNA pair US1+DS2 are able to produce EB that are indistinguishable from the controls (Fig. 1f, Day 0). By day 7 , they give rise to cells that attach to culture plates, indicating that the deletion of FIRE does not affect responsiveness to IL3. By 2 weeks post-differentiation, a lawn of ESC-derived macrophages are produced from the Csfir $1 /+\mathrm{EB}$, whereas the number of macrophages from $C s f 1 r^{\Delta \mathrm{FIRE} / \Delta \mathrm{FIRE}}$ clones was $\approx 200$-fold lower (Fig. 1f). Overall, these in vitro results indicate that FIRE is required for expression of Csf1r and/or macrophage differentiation in EB.

Generation of $\boldsymbol{c s f} 1 \boldsymbol{r}^{\Delta \mathrm{FIRE} / \Delta \mathrm{FIRE}}$ mice. To produce Csf1 $r^{\Delta \text { FIRE/ }}$ FIRE mice, vectors encoding the FIRE gRNAs US1 and DS2 and Cas9 were microinjected into C57BL/6J/CBA F1 mouse oocytes. One male founder with the expected 418 bp deletion was crossed with Csf1r $+1+\mathrm{C} 57 \mathrm{BL} / 6 \mathrm{~J}$ females and the progeny were interbred. The frequencies of $C s f 1 r^{+/+}, C s f 1 r^{+/ \Delta \text { FIRE }}$ and $C s f 1 r^{\triangle \mathrm{FIRE} / \Delta \mathrm{FIRE}}$ mice at weaning are $26 \%, 55 \%$, and $19 \%$, respectively $(n=501)$. Unlike $C s f 1 r^{-1-}$ mice or the Csf1 $1^{o p / o p}$ mouse $^{4}$ there is no postnatal growth retardation, failure of tooth eruption or evidence of osteopetrosis in Csf $1 r^{\Delta \mathrm{FIRE} / \Delta \mathrm{FIRE}}$ mice (Fig. 2a-c). The proximal growth plates of control and Csf1 $r^{\Delta \mathrm{FIRE} / \Delta \mathrm{FIRE}}$ mice are indistinguishable and normal numbers of osteoclasts are present (Fig. 2d, e). Brain abnormalities 
a

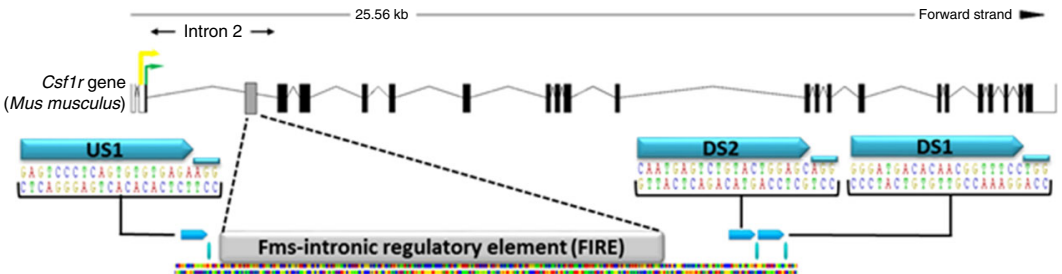

C
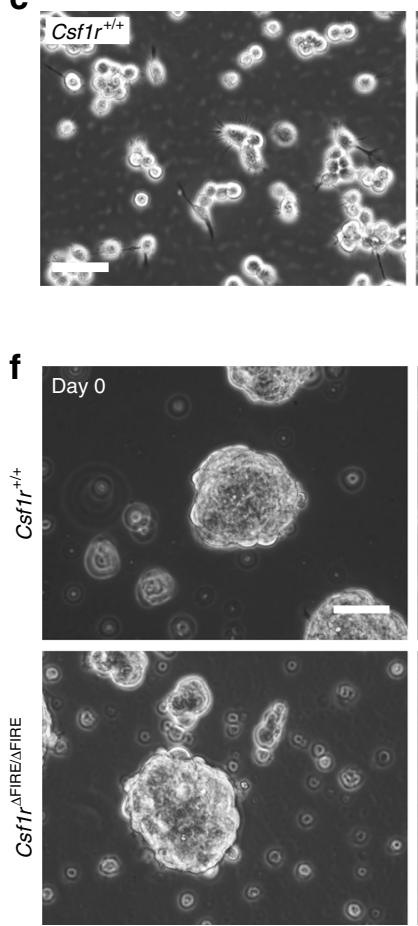
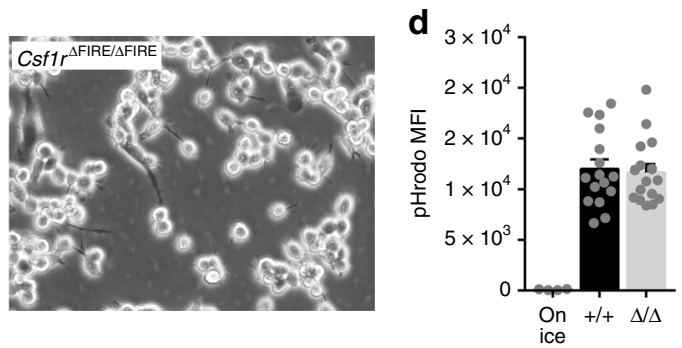

b
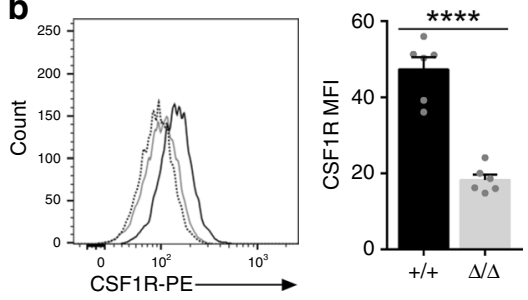

e
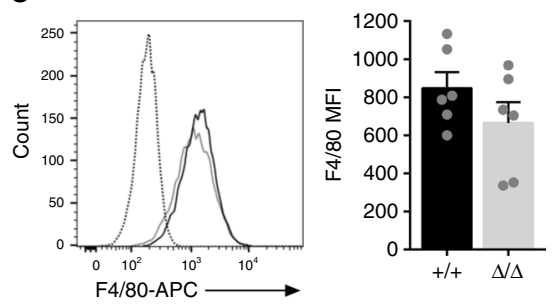

Fig. 1 FIRE deletion reduced CSF1R expression and prevented macrophage differentiation. a Schematic of Csf1r gene and location of gRNAs (US1, DS1, DS2), targeting FIRE. Protospacer adjacent motifs (PAM) are located to the right of each gRNA. Black boxes $=$ coding exons, white boxes $=$ untranslated regions, lines connecting exons = intronic sequences, yellow and green arrows = trophoblast/osteoclast promoter and macrophage transcription start sites,

respectively. $\mathbf{b}$ Flow cytometry analysis of total CSF1R in fixed and permeabilized RAW 264.7 cell clones. Histograms are representative of three clones per genotype, from two repeat experiments in duplicate. MFI median fluorescence intensity, black line $=+/+$, gray line $=\Delta / \Delta$, dotted line $=$ isotype control. $\left.P \leq 0.0001{ }^{(\star \star \star \star}\right)$. c CRISPR-treated Csf1r ${ }^{+/+}$and Csf1r $\Delta$ FIRE/ $\Delta$ FIRE RAW 264.7 cells cultured in vitro. Scale bar $=50 \mu \mathrm{m}$. d A phagocytosis assay was performed with RAW 264.7 cells and pHrodo ${ }^{\oplus}$ Red E. coli BioParticles ${ }^{\oplus}$ followed by flow cytometry analysis. $n=4$ clones per genotype from two repeat experiments. MFI median fluorescence intensity. e Flow cytometry analysis of surface F4/80 in RAW 264.7 cell clones. Histograms are representative of three clones per genotype from two repeat experiments in duplicate. $\mathbf{f}$ Csf1r $+/+$ and Csf1r $\triangle$ FIRE/ $\Delta$ FIRE E14 ESC clones were differentiated into macrophages with mIL3 and rhCSF1. Scale bars $=200 \mu \mathrm{m}$ (Day 0, embryoid bodies) and $50 \mu \mathrm{m}$ (Days 7 and 14). $n=3$ clones per genotype, $P=0.005$ ( ${ }^{\star}$ ). All source data are provided within a Source Data excel file. Graphs show mean + SEM and $P$ values were determined by two-tailed $t$-tests

in $C s f 1 r^{-1-}$ mice include reduced brain size, severely enlarged ventricles, hollow olfactory bulbs, deficient myelination and failure of commissure closing in the corpus callosum ${ }^{19}$. The $C s f 1 r^{\triangle \mathrm{FIRE} / \triangle \mathrm{FIRE}}$ brains are not macroscopically distinguishable from controls and Luxol fast blue staining reveals no differences in myelination (Fig. 2f, g).

Csf $1^{\mathrm{op} / \mathrm{op}}$, Csf $1 \mathrm{r}^{-{ }^{-}}$mice and adult mice treated with antiCSF1R antibody lack lamina propria and Peyer's patch macrophages in the small intestine. These mice also show associated changes in villus architecture, increased goblet cell numbers detected with periodic acid-schiff (PAS) staining, reduced proliferation in the crypts and defects in differentiation of Paneth cells $s^{6,20,21}$. Csf1r mRNA is not expressed in intestinal epithelial cells and the impacts of anti-CSF1R treatment are attributable to the loss of macrophages surrounding the crypts ${ }^{21}$. There are no detectable alterations in intestinal epithelia that distinguish between $C s f 1 r^{+/+}$and $C s f 1 r^{\Delta \text { FIRE/ }} \Delta$ FIRE mice (Fig. $2 \mathrm{~h}$ and Supplementary Fig. 2).

The development of normal sexual maturity is compromised in both male and female Csf $1^{\text {op/op }}$ mice 22,23 and in Csf $1 r^{-1-}$ rats $^{5}$. By contrast, the $C s f 1 r^{\Delta \text { FIRE/ }}$ FIRE mice reach sexual maturity and are fertile.

The impact of FIRE deletion in blood and BM. Two subsets of blood monocytes can be distinguished based upon the expression of LY6C. The differentiation of the LY6Clo subset depends upon CSF1R signaling ${ }^{6,24}$. Csf $1 r^{\triangle F I R E / \Delta F I R E ~ m i c e ~ h a v e ~ n o r m a l ~ c e l l ~}$ populations in peripheral blood (Fig. 3a) and F4/80, LY6C, and $\mathrm{CD} 11 \mathrm{~b}$ expression is unchanged compared to littermates (Fig. 3b-d). However, the expression of CSF1R detected with anti-CD115 antibody is abolished (Fig. 3c). Furthermore, the binding of labeled CSF1 (pCSF1-Fc $\left.{ }^{\mathrm{AF} 647}\right)^{11}$ is undetectable in the mutant mice, whereas in littermates it provides a sensitive marker 

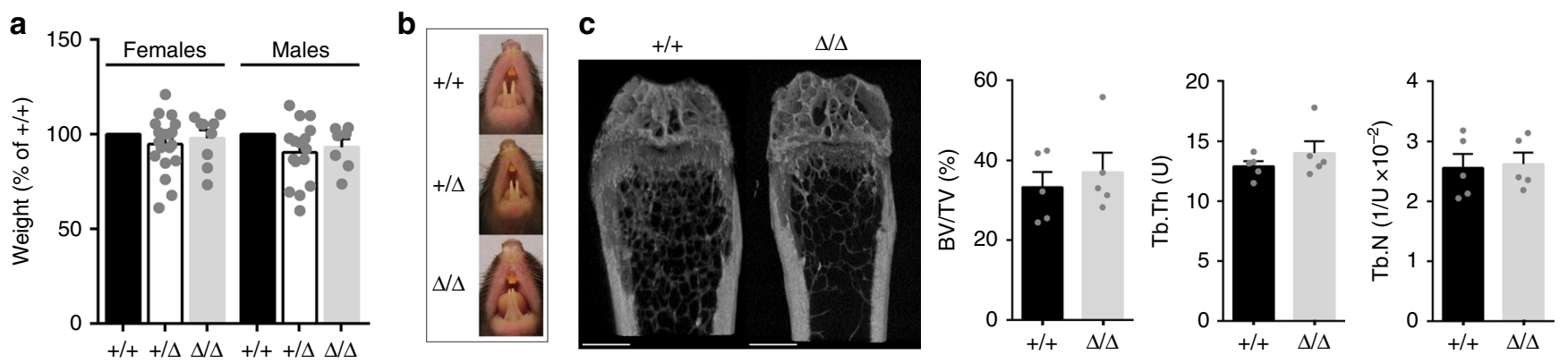

d

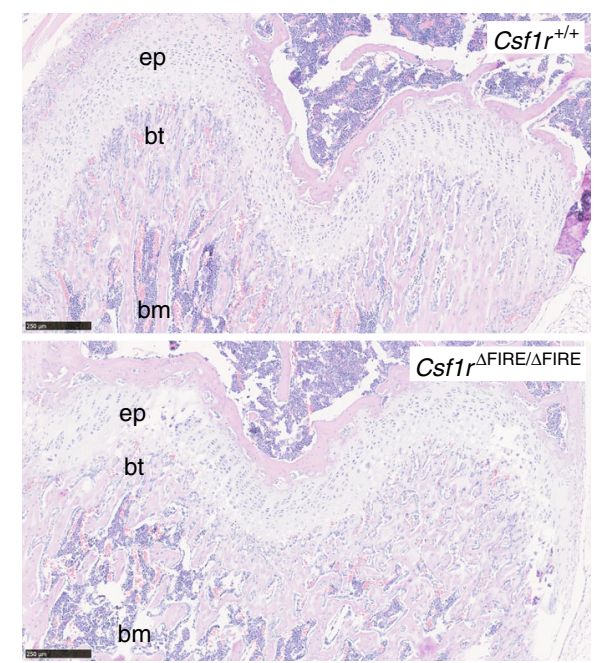

e

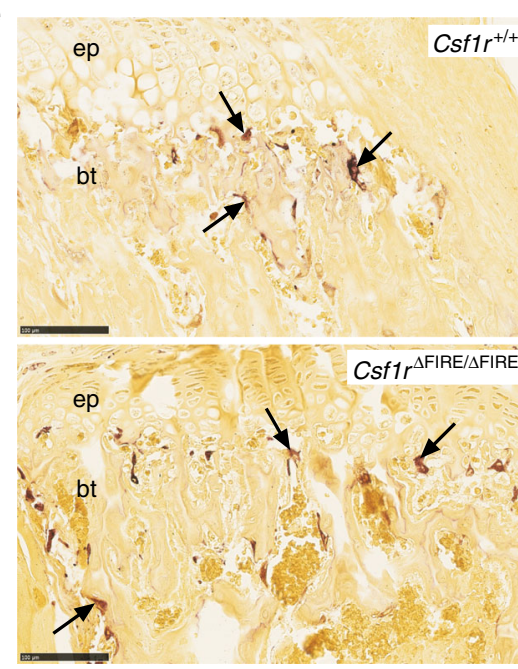

f

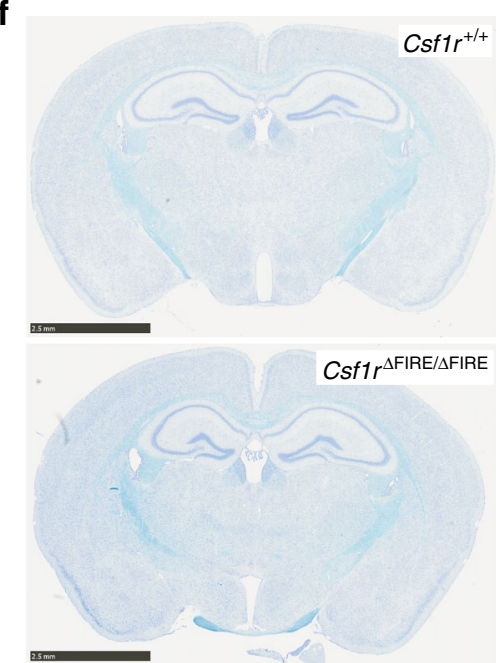

g

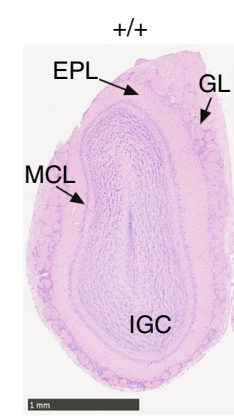

$\Delta / \Delta$

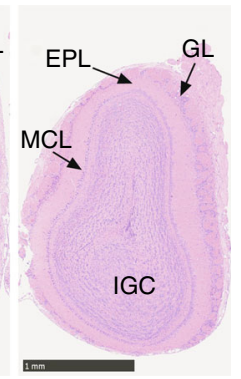

h

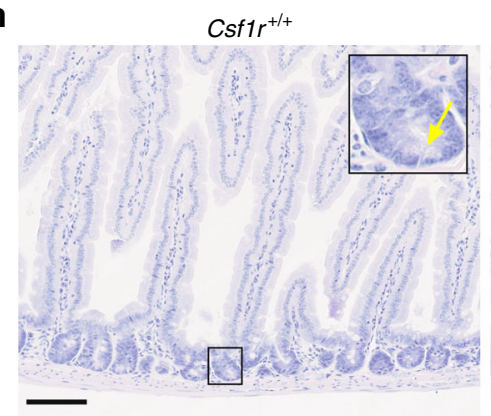

Csf1r $\triangle \mathrm{FIRE/AFIRE}$

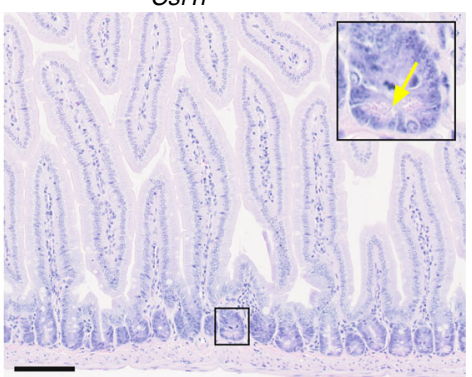

Fig. 2 FIRE-deficient mice displayed none of the gross phenotypes found in Csf1r ${ }^{-/}{ }^{-}$-mice. a Mice were weighed between 2 and 11 weeks of age. $n=7,20$, 9 (females) and 5, 16, 7 (males) for $+/+,+/ \Delta$, and $\Delta / \Delta$, respectively. b Representative images of tooth eruption in adult mice. $\mathbf{c}$ Femurs from 4-week-old male and female mice were scanned by Micro-CT. Scale bars $=1 \mathrm{~mm} . n=5$ mice per genotype. BV/TV bone volume over total volume (bone density), Tb. Th trabecular thickness, Tb.N trabecular number. d Hematoxylin and eosin (H\&E) staining of sections of bone. Images are representative of six mice per genotype; ep epiphyseal plate, bt bony trabeculae, bm bone marrow. Scale bar $=250 \mu \mathrm{m}$. e Femurs from the same cohort of mice above were stained for tartrate-resistant acid phosphatase (TRAP). Arrows point to TRAP + osteoclasts. Scale bar $=100 \mu \mathrm{m}$. $\mathbf{f}$ Luxol Fast Blue staining of paraffin-embedded formalin-fixed adult brains. Images are representative of three mice per genotype. Scale bar $=2.5 \mathrm{~mm}$. $\mathbf{g}$ H\&E staining of olfactory bulbs from adult mice. Images are representative of six mice per genotype. EPL external plexiform layer, GL glomerular layer, IGC internal granular cell layer of the olfactory bulbs, MCL mitral cell layer. Scale bar $=1 \mathrm{~mm}$. $\mathbf{h}$ H\&E staining of sections of duodenum from adult mice. Yellow arrows point to Paneth cells. Images are representative of six mice per genotype. Scale bar $=100 \mu \mathrm{m}$. All source data are provided within a Source Data excel file. Graphs show mean $+\mathrm{SEM}$

for $\mathrm{F} 4 / 80^{+}$blood monocytes (Fig. 3d). Notably, the level of both CSF1R and CSF1 binding is reduced by around $50 \%$ in the heterozygotes (Fig. 3c, d), which is consistent with a lack of dosage compensation at the mRNA level also reported in Csf1r-deficient mice and rats ${ }^{4,5}$.

Unlike Csf1r and Csf1 mutant mice ${ }^{4}, C s f 1 r^{\Delta \text { FIRE/ }}$ FIRE mice are not osteoclast-deficient and osteopetrotic. Total BM cellularity remains unchanged by the FIRE mutation (Supplementary Fig. 3a). Aside from progenitors, BM contains multiple specialized macrophage populations that regulate bone homeostasis and hematopoietic differentiation (reviewed in ref. ${ }^{25}$ ). BM cell populations were analyzed by flow cytometry as described in Supplementary Fig. 3b. Two populations of $\mathrm{F} 4 / 80^{+}$cells are detected in control mice that differed in the level of CSF1R. The proportion of F4/80+ cells is unchanged in Csfir $r^{\Delta \mathrm{FIRE} / \Delta \mathrm{FIRE}}$ mice, but CSF1R expression and CSF1 binding is abolished in both $\mathrm{F} 4 / 80^{+}$populations (Supplementary Fig. $3 c$ and d). As in the blood, the level of CSF1R is reduced around $50 \%$ in heterozygotes. BM contains a population of monocyte-dendritic cell progenitors (MDP) that express KIT, FLT3, and CSF1R, and committed proliferative $\mathrm{LY}^{+}$progenitors derived from the MDP that lack both KIT and FLT3 ${ }^{26}$. Although CSF1R expression is lost in Csfl $\triangle \mathrm{FIRE} / \Delta \mathrm{FIRE} \mathrm{BM}$, there is no 

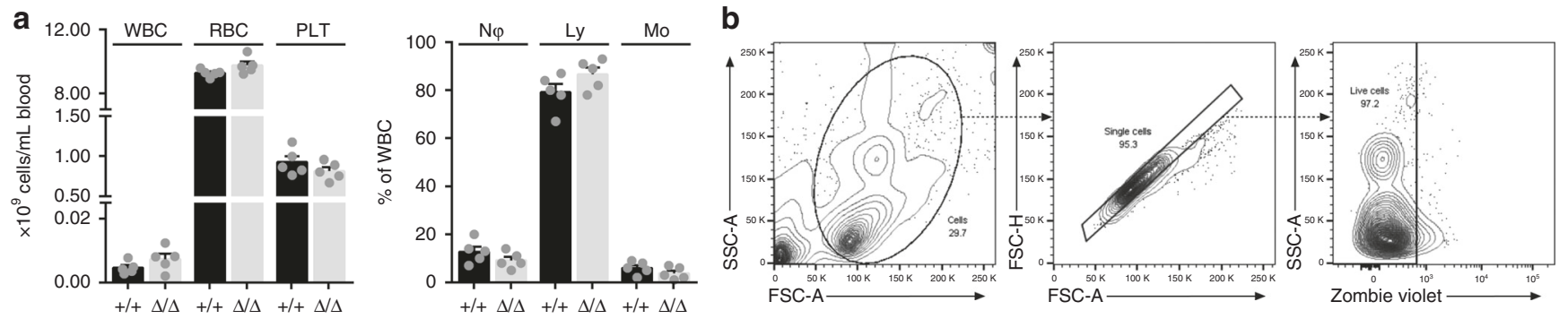
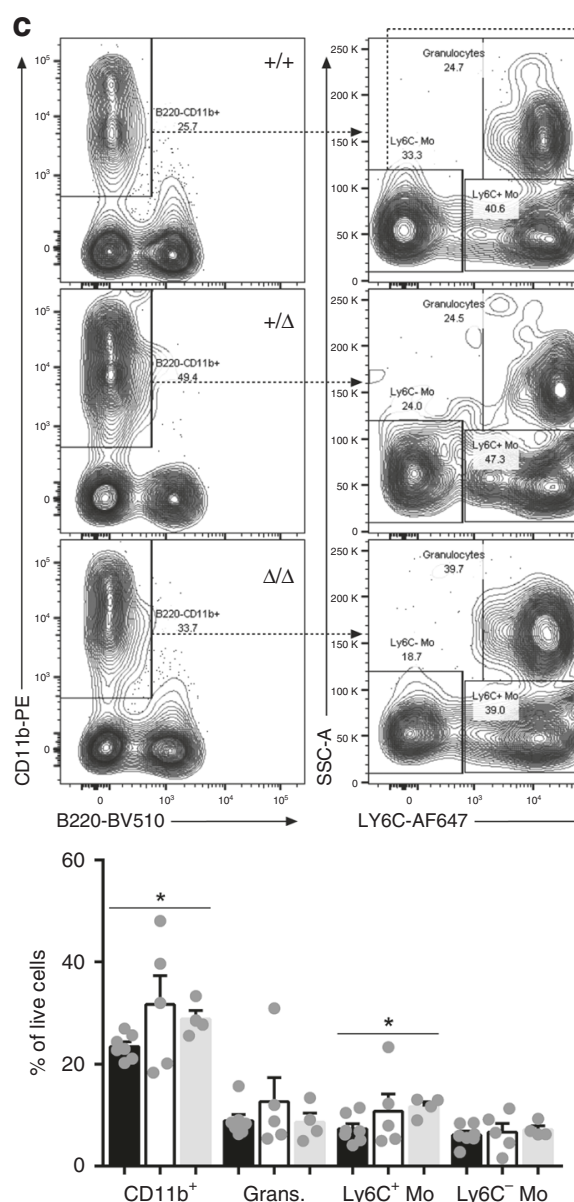
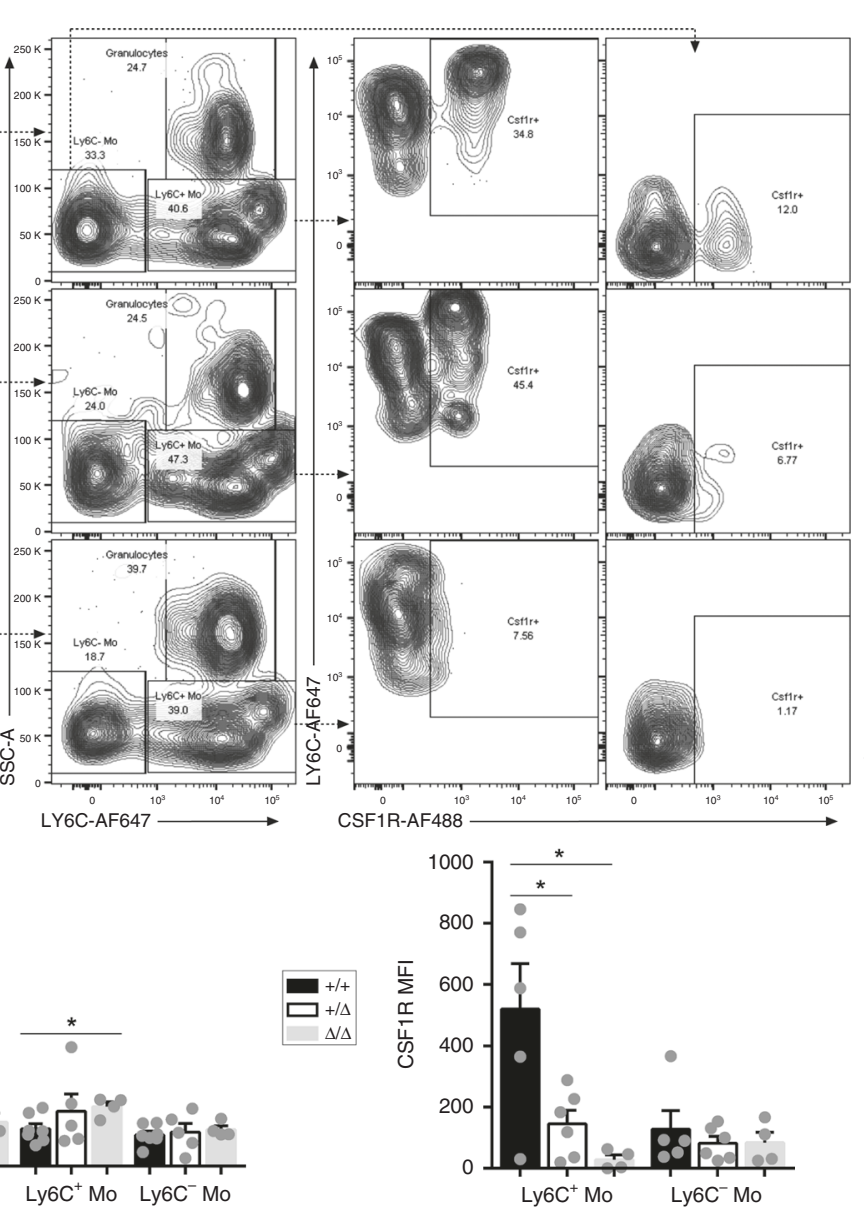

d
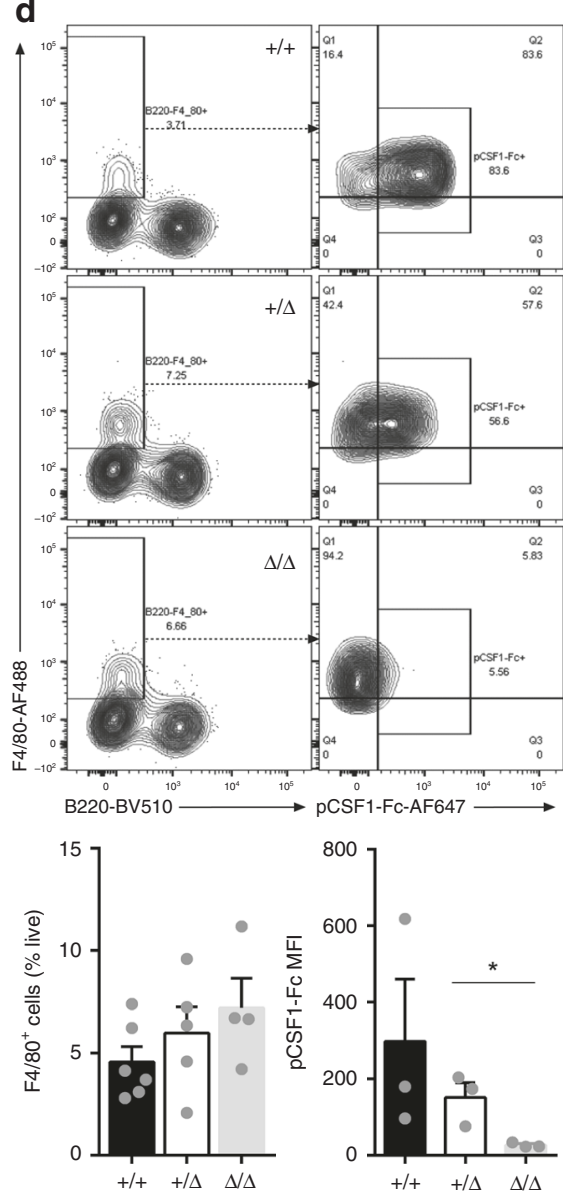

Fig. 3 FIRE deletion reduced functional CSF1R expression in blood. a Cell populations in whole blood of 11-13-week-old Csf1r $\Delta$ FIRE/ $\Delta$ FIRE mice $(\Delta / \Delta)$ and controls $(+/+)$ were quantified using an automated hematology analyzer. $n=5$ mice per genotype. WBC white blood cells, RBC red blood cells, PLT platelets, N $\varphi$ neutrophils, Ly lymphocytes, Mo monocytes. b Whole EDTA-blood from 11 to 13 -week-old mice was analyzed by flow cytometry. Plots show the representative gating strategy to identify live single cells for subsequent analysis. c Myeloid cells were identified as CD11b+B220- (Panel 1) then separated by LY6C and SSC to identify granulocytes ( $\mathrm{LY}_{6 \mathrm{C}}{ }^{+} \mathrm{SSC}$ hi, panel 2). The $\mathrm{LY} 6 \mathrm{C}^{+}$and LY6Clo monocyte populations were then analyzed for CSF1R expression (Panels 3 and 4 , respectively). $n=7+/+, 5+/ \Delta$, and $4 \Delta / \Delta$ from four experiments. For $\%$ of live cells $P=0.012\left({ }^{\star} \mathrm{CD} 11 \mathrm{~b}^{+}\right)$and $0.019\left({ }^{\star} \mathrm{LY} 6 \mathrm{C}^{+}\right.$ Mo). For median fluorescence intensity (MFI) of CSF1R $P=0.027\left({ }^{*}+/+\right.$ vs. $\left.+/ \Delta\right)$, and $0.022\left({ }^{*}+/+\right.$ vs. $\left.\Delta / \Delta\right)$. Grans. granulocytes, Mo monocyte. d F4/ $80^{+} \mathrm{B}_{22} 0^{-}$myeloid cells were analyzed for binding of CSF1 (pCSF1-Fc $\mathrm{AF647}^{11}$. $n=3$ mice per genotype from four experiments. $P=0.033\left({ }^{*}\right.$ ). All source data are provided within a Source Data excel file. Graphs show mean + SEM and $P$ values were determined by two-tailed $t$-tests

reduction in the number of cells expressing LY6C, KIT, or FLT3 (Supplementary Fig. 3e). In view of the apparent loss of CSF1R protein, we examined whether $\mathrm{BM}$ from mutant mice is responsive to CSF1. BM-derived macrophages (BMDM) are generated from $C s f 1 r^{\triangle F I R E / \triangle F I R E} B M$, but with greatly reduced yield compared to controls (Supplementary Fig. 3f). The macrophages derived from $C s f 1 r^{\Delta \text { FIRE/ } \triangle \text { FIRE }}$ BM still express F4/80 and CD11b but have lower levels of surface CSF1R detected with pCSF1-Fc ${ }^{\mathrm{AF} 647}$ relative to controls (Supplementary Fig. 3g). Hence, the production/survival of these few BMDM relies on some mechanism that compensates for the absence of FIRE.
Macrophage populations in $\boldsymbol{C s f 1} \boldsymbol{r}^{\Delta \mathrm{FIRE} / \Delta \mathrm{FIRE}}$ mice. We performed flow cytometry and/or immunohistochemistry analyses on major organs as shown in Fig. 4, Supplementary Figs. 4 and 5. Liver macrophages (Kupffer cells, KC) are CSF1R-dependent ${ }^{4,6}$, CSF1-responsive ${ }^{27}$, and the main site of clearance of labeled CSF $1^{11}$. The number, location, or morphology of $\mathrm{KC}$ and the liver-to-body weight ratio of $C s f 1 r^{\triangle \mathrm{FIRE} / \Delta \mathrm{FIRE}}$ mice are similar to littermate controls (Supplementary Fig. 4a, b). Flow cytometry analysis confirmed the unchanged numbers of $\mathrm{KC}$ and dendritic cell subsets (cDC2 as defined previously ${ }^{11}$ ) (Fig. 4a) and retention of KC-specific markers such as TIM4 (Supplementary Fig. 4c). 
a
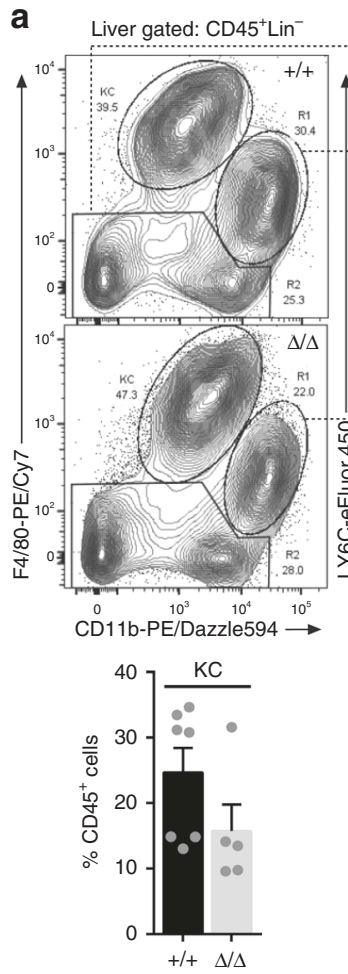
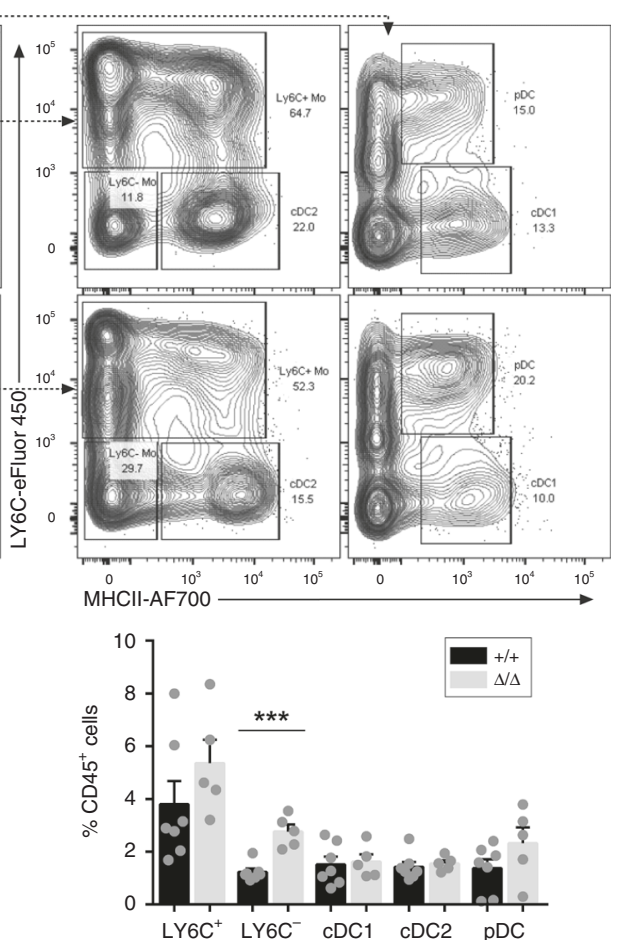

b

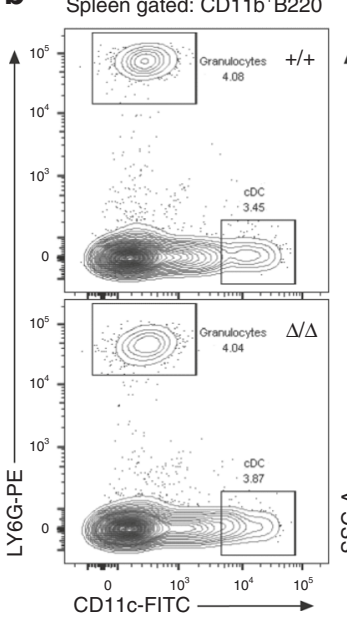

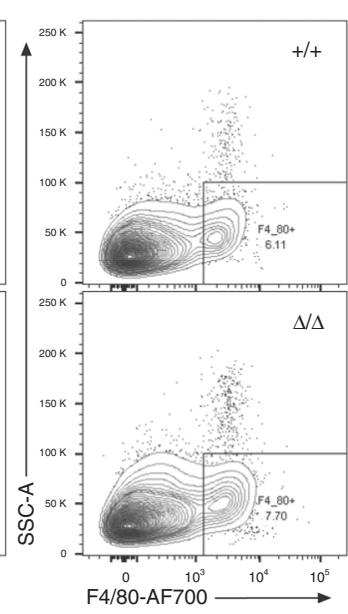

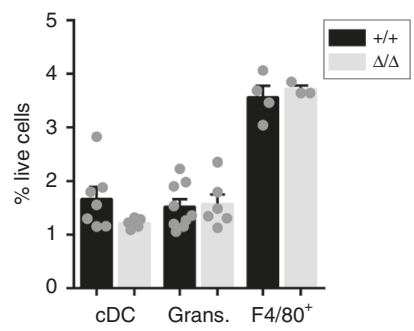

c
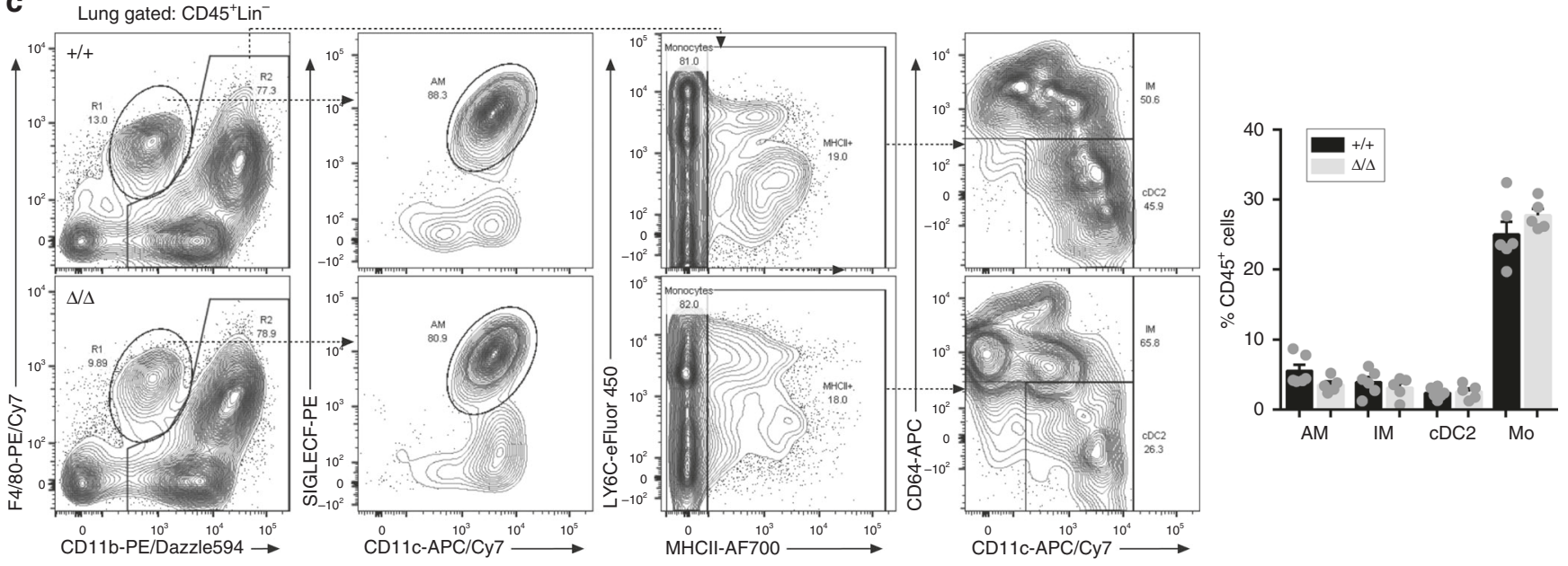

Fig. 4 Macrophage populations in liver, spleen, and lungs are unaffected in FIRE-deficient mice. a Leukocyte populations were analyzed from enzymatically digested livers from mice aged between 8 and 10 weeks. Flow cytometry profiles show representative $\operatorname{Csf1r}+/+(+/+)$ and $\operatorname{Csf1r\Delta FIRE/\Delta FIRE}(\Delta / \Delta)$ mice using the gating strategy described by Hawley et al. ${ }^{11}$. Kupffer cells (KC) were identified as CD45+, lineage ${ }^{-}\left(\right.$Lin-; $^{-}$CD3 $^{-}$CD19- $^{-}$LY6G $^{-}$) and F4/ $80^{\text {hi }} \mathrm{CD} 11 \mathrm{~b}^{\text {lo }}$ (Panel 1). F4/80 lo $/ \mathrm{CD} 11 \mathrm{~b}^{\text {hi }}$ and $\mathrm{F} 4 / 80^{-} / \mathrm{CD} 11 \mathrm{~b}^{-}$populations were further separated on the basis of LY $6 \mathrm{C}$ and MHCII expression (Panels 2 and 3). Mo monocyte, $C D C$ conventional dendritic cell, $\mathrm{pDC}$ plasmacytoid dendritic cell. $n=7+/+$ and $5 \Delta / \Delta$ from 3 repeat experiments. $P=0.0002$ $\left.{ }^{(\star \star \star}\right)$. b Leukocyte populations were analyzed from enzymatically digested spleens from mice aged between 8 and 10 weeks. The flow cytometry profiles

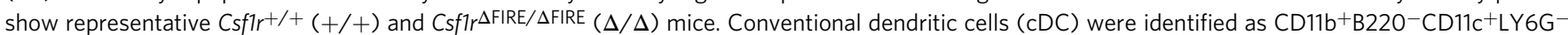
and granulocytes as CD11b+B220-CD11 ${ }^{-} \mathrm{LY} \mathrm{GG}^{+}$(Panel 1). Red pulp macrophages were identified as CD11b ${ }^{+} \mathrm{B} 220^{-} \mathrm{F} 4 / 80^{+} \mathrm{SSClo}(\mathrm{Panel} 2) . n=3-9$ mice per genotype from 4 repeat experiments. c Single cell suspensions of digested lungs were analyzed by flow cytometry as described above for the liver using

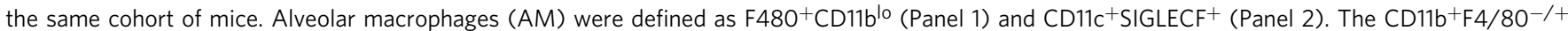

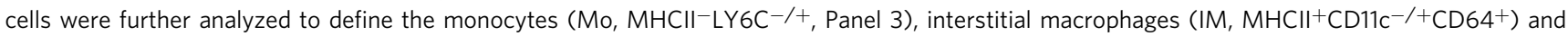
conventional dendritic cells subset $2\left(\mathrm{cDC} 2, \mathrm{MHClI}^{+} \mathrm{CD} 11 \mathrm{c}^{+} \mathrm{CD} 64^{-}\right)$in Panel 4. All source data are provided within a Source Data excel file. Graphs show mean + SEM and $P$ values were determined by two-tailed $t$-tests

However, there is a significant increase in LY6C lo monocyte-like cells $\left(\mathrm{CD} 11 \mathrm{~b}^{+} \mathrm{F} 4 / 80^{\mathrm{lo}}\right)$. The full gating strategy for liver flow cytometry data is shown in Supplementary Fig. $4 \mathrm{~d}$.

The marginal zone is completely absent in the spleen of Csf1 $1^{\text {op }} / \mathrm{pp}$ mice $^{28}$ and in $C s f 1 r^{-1-}$ rats $^{5}$. In Csfl $r^{\Delta \mathrm{FIRE} / \Delta \mathrm{FIRE}}$ mice the overall architecture and relative area of the marginal zone is indistinguishable from the controls (Supplementary Fig. 4e and f). As in the liver, there is no change in the spleen-to-body weight ratio or the relative abundance of myeloid populations defined by staining for $\mathrm{F} 4 / 80^{+}$or other markers (Supplementary Fig. $4 \mathrm{~g}$, $\mathrm{h}$ and Fig. $4 \mathrm{~b}$ ). The full gating strategy for spleen flow cytometry data is shown in Supplementary Fig. 4 i. 
Lung macrophage populations are primarily controlled by CSF2 (also known as GM-CSF, encoded by Csf2), rather than $\mathrm{CSF}^{29}$, but lung macrophages do express Csf1r and bind CSF $1^{11}$. The Csf1r-EGFP macrophage-reporter transgene is highly expressed in both alveolar (AM) and interstitial macrophage populations in the lung ${ }^{10}$, which have distinct developmental origins and renewal mechanisms during adulthood ${ }^{30}$. Furthermore, expression of Csf1r-EGFP in AM is dependent upon the inclusion of FIRE in the transgene ${ }^{10}$. Macrophages of the lung are partly depleted in $C s f 1^{o p / o p}$ mice $^{31}$ and double mutant $C s f 1^{\circ p / o p}$ and $C s f 2^{-1-}$ mice develop severe alveolar proteinosis ${ }^{29}$. AM can be identified based upon expression of SIGLECF ${ }^{30}$. Interstitial myeloid populations have been classified as macrophage and conventional dendritic cell subpopulations, based upon expression of CD64, CD11b, CD11c, and MHCII ${ }^{11}$. To assess the impact of the FIRE deletion on these populations, lungs were digested and analyzed by flow cytometry as previously described ${ }^{11}$. The full gating strategy is shown in Supplementary Fig. 5a. We also measured Csflr mRNA in the lung of wild-type

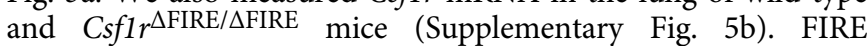
deletion has no effect on AM or any of the interstitial myeloid populations of the lung (Fig. 4c). A marginal reduction in Csf1r mRNA is likely attributable to the loss of expression in monocytes in both blood and BM (Fig. 3c, d and Supplementary Fig. 3c, d). F4/80 is barely detectable in either of the major lung macrophage populations and is primarily associated with monocytes ${ }^{30}$. Accordingly, the levels of total Adgre1 (encoding F4/80) and Itgam (CD11b) mRNA in the lung are unaffected by the deletion of FIRE. We conclude that FIRE is not required for expression of Csflr mRNA in lung macrophages nor for their development or monocyte trafficking in the lung.

The large macrophage population of the intestine is constantly renewed from recruited blood monocytes ${ }^{32}$. The Peyer's patch and surrounding mucosa contain several specialized mononuclear phagocyte populations, distinguished by mRNA or protein expression of the surface markers F4/80 (ADGRE1), CD4, SIGLEC1, SIRPA, MERTK, TIM4, CX3CR1, and ITGAX $(\mathrm{CD} 11 \mathrm{c})^{33}$, including a recently described long-lived population ${ }^{34,35}$. All of these populations depend upon continuous CSF1R signaling ${ }^{6,21}$. Monocytes enter the lamina propria constantly and respond to the local environment with progressive changes in surface phenotype and gene expression in a so-called monocyte waterfall ${ }^{32}$. Supplementary Fig. 6 shows the compar-

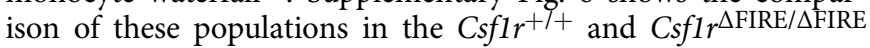
mice. There is no detectable difference in distribution of monocyte-like, DC-like, or macrophage populations in either small or large intestine.

Macrophages are so abundant in the intestine that the level of macrophage-specific transcripts can be reproducibly detected by analysis of total mRNA. To complement the analysis of isolated cells, we isolated mRNA from Peyer's patches and surrounding mucosa in the ileum of Csf1r ${ }^{+/+}$and $C s f 1 r^{\Delta \mathrm{FIRE} / \Delta \mathrm{FIRE}}$ mice and profiled gene expression using microarrays. There is no significant difference in Csf1r expression between Csf1r ${ }^{+/+}$and $C s f 1 r^{\triangle F I R E / \triangle F I R E}$ mice nor in the level of mRNA encoding any of the markers of intestinal macrophage subpopulations mentioned above, including Itgam (CD11b) and $L y 6 c 1$, which are rapidly down-regulated following extravasation into the intestine ${ }^{32}$ (Supplementary Data 1). These findings demonstrate that FIRE is not required by intestinal macrophages for expression of Csf1r and that the loss of CSF1R in monocytes has no impact on intestinal macrophage homeostasis. In addition, the expression of transcripts that mark cell types controlled indirectly by signals from CSF1R-dependent macrophages, including markers of Paneth cells (Lyz1), microfold cells (Spib, Ccl9, Tnfrsf11a) or goblet cells $(M u c 2)^{21}$, are unaffected (Supplementary Data 1).

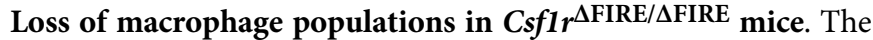
resident macrophages of the peritoneal cavity may be subdivided into two populations, based upon relative size and the level of TIM4 and F4/80 antigen they express ${ }^{36}$. Both populations of peritoneal macrophages (PM) are absent from Csf1 $1^{o p / o p}$ and Csf $1 r^{-1-}$ mice ${ }^{4,28}$ and from mice treated with anti-CSF1R antibody ${ }^{6}$. The major TIM $4^{+} \mathrm{F} 4 / 80^{\text {hi }} \mathrm{CD} 11 \mathrm{~b}^{\text {hi }}$ large $\mathrm{PM}$ population is almost entirely lost in Csf1r $r^{\triangle \mathrm{FIRE} / \Delta \mathrm{FIRE}}$ mice (Fig. 5a and Supplementary Fig. 6a). The minor residual PM population consists of the small PM that are TIM4-F4/80 ${ }^{\text {lo36 }}$. These cells were shown previously to bind labeled CSF1 preferentially compared to large $\mathrm{PM}^{11}$. Both anti-CSF1R (CD115) and CSF1-Fc binding to these residual populations are absent in the Csf $1 r^{\Delta \mathrm{FIRE} / \Delta \mathrm{FIRE}}$ mice (Fig. 5a). In Csflr $r^{\Delta \mathrm{FIRE} /+}$ mice the PM populations are retained but as in blood and BM, there is a reduction in the level of surface receptor detected with either labeled CSF1 or anti-CSF1R antibody, which is consistent with the lack of dosage compensation. In summary, in the peritoneum, the $C s f 1 r^{\Delta \mathrm{FIRE} / \Delta \mathrm{FIRE}}$ mutation phenocopies the Csflr-deficient mice.

The abundant interstitial F4/80 hi macrophage populations of the kidney have recognized roles in renal growth and development ${ }^{37}$, homeostasis and immune surveillance ${ }^{38}$. F4/80 hi kidney macrophages were almost absent from both $C s f 1^{\text {op/op }}$ and $C s f 1 r^{-/-}$mice ${ }^{4}$. To identify populations of renal mononuclear phagocytes we enzymatically digested kidneys and analyzed $\mathrm{CD} 45^{+}$myeloid cells by flow cytometry. The gating strategy is shown in Supplementary Fig. 7b. Csf $1 r^{\Delta \text { FIRE } / \Delta \text { FIRE }}$ mice have fewer total $\mathrm{CD} 45^{+}$cells and the $\mathrm{F} 4 / 80^{\text {hi }} \mathrm{CD} 11 \mathrm{~b}^{\text {lo }}$ resident kidney macrophages $(\mathrm{KM})^{38}$ are undetectable (Fig. 5b). In both control and mutant, a subset of the CD11b hi cells are F4/

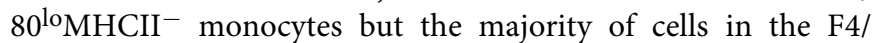
$80^{\mathrm{lo}} \mathrm{CD} 11 \mathrm{~b}^{\text {hi }}(\mathrm{R} 1)$ gate are also $\mathrm{MHCII}^{+} / \mathrm{CD} 4^{+}$. Their surface phenotype is reminiscent of the monocyte-derived small PM discussed above. As in the peritoneum, their abundance is slightly increased by the FIRE mutation (Fig. 5b). Conventional dendritic cells (cDC) in the kidney are difficult to separate from macrophages based upon surface markers. A minor subpopula-

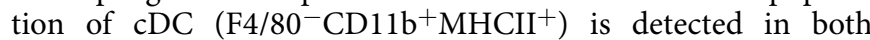
control and $C s f 1 r^{\Delta \mathrm{FIRE} / \Delta \mathrm{FIRE}}$ kidneys (R2 gate) (Fig. 5b). Although exogenous CSF1 can promote mouse kidney morphogenesis in vitro and growth of the kidney in vivo ${ }^{37}$, there is no change in the kidney-to-body weight ratio in $C s f 1 r^{\Delta F I R E / \Delta F I R E ~}$ mice (Supplementary Fig. 7c) and kidneys are histologically normal (Supplementary Fig. 7d).

Macrophages of the heart can also be separated into subpopulations attributed distinct roles in cardiac homeostasis and repair following injury ${ }^{39-41}$. Supplementary Fig. 7e shows the flow cytometry gating strategy for the heart and Fig. 5c summarizes the analysis of digested cardiac macrophage populations from control and Csf $1 r^{\Delta \mathrm{FIRE} / \Delta \mathrm{FIRE}}$ mice. The total cardiac $\mathrm{CD} 45^{+}$population is greatly reduced in the $C s f 1 r^{\Delta \mathrm{FIRE} / \Delta \mathrm{FIRE}}$ mice due to selective loss (70-80\%) of both the $\mathrm{F} 4 / 80^{\mathrm{hi}} \mathrm{CD} 64^{+}$ macrophage $\left(\mathrm{MHCII}^{\mathrm{hi}}\right)$ and monocyte-like $\left(\mathrm{MHCII}^{\mathrm{lo}}\right)$ populations previously described by others ${ }^{39-41}$. Interestingly, a recent study proposed a link between resident macrophages of the kidney and the heart in the generation of an adaptive response to cardiac pressure overload ${ }^{40}$. However, as in the kidney, we detect no change in the heart-to-body weight ratio in Csf1r $\Delta \mathrm{FIRE} / \Delta \mathrm{FIRE}$ mice (Supplementary Fig. 7f) and the histology is indistinguishable from controls (Supplementary Fig. 7g).

There are two major macrophage populations in the skin, the Langerhans cells of the epidermis and the dermal macrophages. Both are CSF1R-dependent ${ }^{4,6}$ but Langerhans cells, like microglia, depend specifically on the alternative CSF1R ligand, IL34 ${ }^{42}$. Langerhans cells are absent in epidermal sheets from tails of Csfir $\triangle$ FIRE/ $\triangle$ FIRE mice (Fig. 6a). The loss of this cell population 
a Peritoneal cavity gated: live
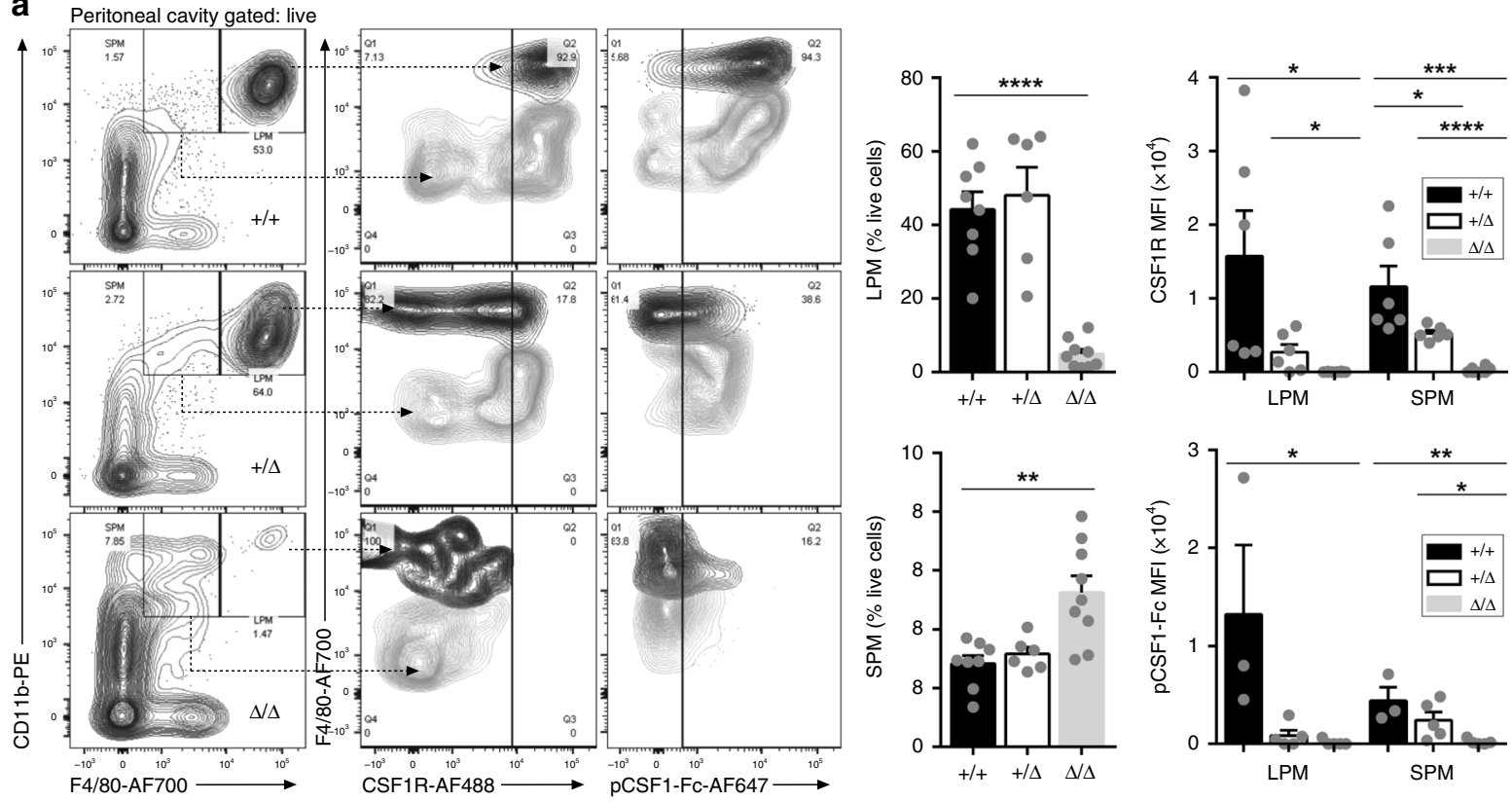

b Kidney gated: $C D 45^{+}$
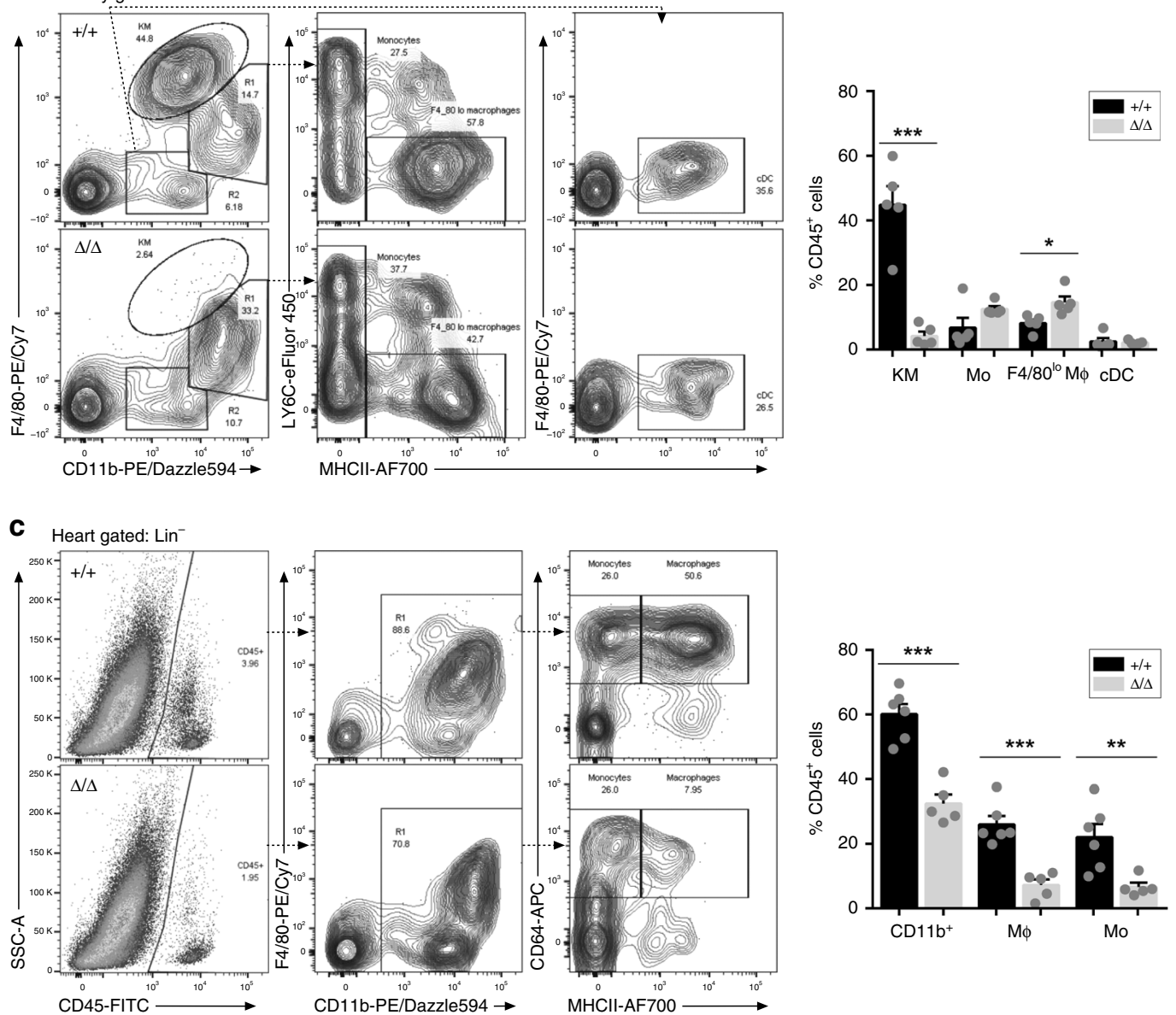

has no effect on the morphology of the epidermis or the dermis (Fig. 6b). Dermal macrophage populations accumulate melanosomes from neighboring melanocytes and, like macrophages of the gut, they turn over rapidly ${ }^{43}$. The stellate melanophagic macrophage populations of the dermis are $\mathrm{F} 4 / 80^{+}$and are not affected by the FIRE mutation (Fig. 6b).
Loss of microglia in Csfl $r^{\Delta \mathrm{FIRE} / \Delta \mathrm{FIRE}}$ mice. Microglia, the tissue-resident macrophage population of the brain, arise very early in mouse embryonic development and depend upon CSF1R signaling ${ }^{44}$. Microglia detected by localization of IBA1 or P2RY12 in littermate controls are absent in the brain of Csfl $r^{\triangle \mathrm{FIRE} / \Delta \mathrm{FIRE}}$ mice (Fig. 7a, b). Disaggregation of the whole 
Fig. 5 FIRE deletion results in loss of macrophages in the peritoneal cavity, kidney, and heart. a Flow cytometry profiles show peritoneal cells from representative $C s f 1 r^{+/+}$and $C s f 1 r^{\Delta F I R E / \Delta F I R E}$ mice using the gating strategy described by Bain et al. ${ }^{36}$. Small peritoneal macrophages (SPM) were identified as $\mathrm{F} 4 / 80^{\circ} \mathrm{CD} 11 \mathrm{~b}^{+}$and large peritoneal macrophages (LPM) as F4/80hi CD11b+ (Panel 1). These populations were analyzed for CSF1R expression (Panel 2) and binding of pCSF1-Fc (Panel 3). $n=8+/+, 6+/ \Delta$ and $9 \Delta / \Delta$ mice aged between 10 and 15 weeks, from five experiments. $P=0.012-0.048\left({ }^{*}\right)$, $\left.0.002-0.006^{(\star \star}\right), 0.001\left(^{\star \star \star}\right)$ and $<0.0001\left(^{\star \star \star \star}\right)$. b Leukocyte populations were isolated from enzymatically digested kidneys and gated for CD45 expression. The flow cytometry profiles show analysis of representative Csf1r $+/+$ and $C s f 1 r^{\Delta F I R E / \Delta F I R E}$ mice. Kidney macrophages (KM) were identified as

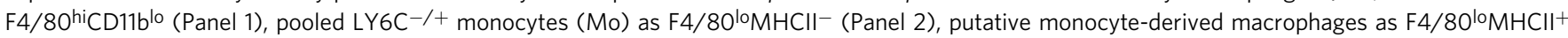
(Panel 2), and conventional dendritic cells ( $\mathrm{CDC}$ ) as $\mathrm{F} 4 / 80^{-} \mathrm{CD} 11 \mathrm{~b}^{\mathrm{l}} \mathrm{MHCl}+\mathrm{H}^{+}$(Panel 3). $n=5$ mice per genotype aged 8-10 weeks, from three experiments. $P=0.015^{(*)}$ and $0.0001\left(^{\star * \star}\right)$. c Single cell suspensions of enzymatically digested hearts from the same cohort were analyzed by flow cytometry. Cells were gated lineage ${ }^{-}\left(\mathrm{Lin}^{-}=\mathrm{CD} 3 / \mathrm{CD} 19 / \mathrm{LY} 6 \mathrm{G}\right) . n=6+/+$ and $5 \Delta / \Delta$ from three experiments. M $\Phi$ macrophage, Mo monocyte. $P=0.009\left({ }^{\star}\right), 0.0003$ $\left({ }^{\star \star \star} \mathrm{M} \Phi\right)$, and $\left.0.0001{ }^{\star \star \star} \mathrm{CD} 11 \mathrm{~b}^{+}\right)$. All source data are provided within a Source Data excel file. Graphs show mean $+\mathrm{SEM}$ and $P$ values were determined by two-tailed $t$-tests
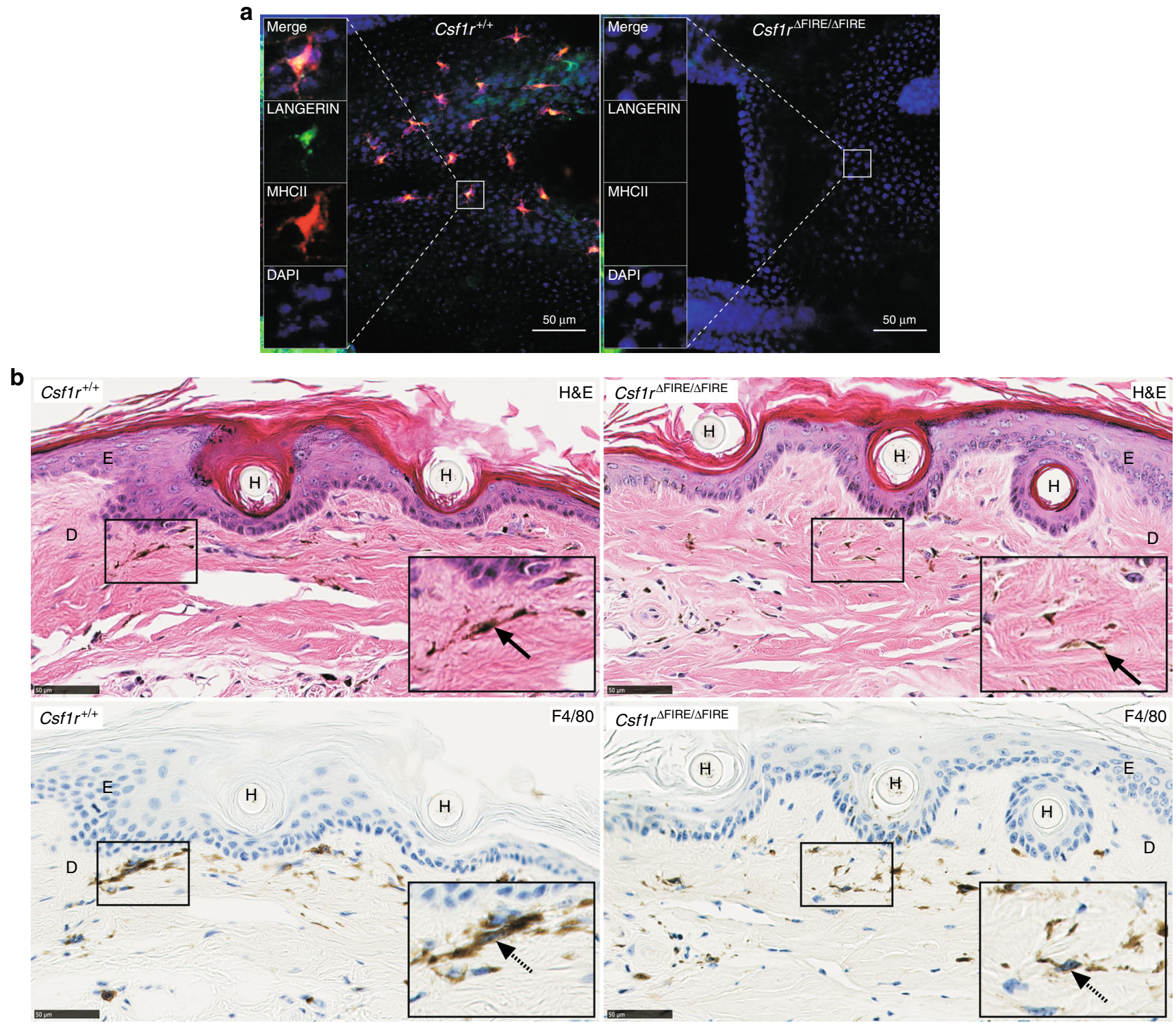

Fig. 6 The deletion of FIRE results in loss of Langerhans cells. a Whole-mount epidermal tail sheets were stained with antibodies against LANGERIN (green) and $\mathrm{MHCll}$ (red). Nuclei were stained with DAPI (blue). Images are representative of four mice per genotype, aged between 8 and 10 weeks. Scale bars $=50 \mu \mathrm{m}$. b Decalcified tails from the same cohort of mice above were stained with hematoxylin and eosin (H\&E, upper panel) and an antibody against F4/80 (lower panel). E epidermis, D dermis, H hair follicle. Scale bars $=50 \mu \mathrm{m}$ 

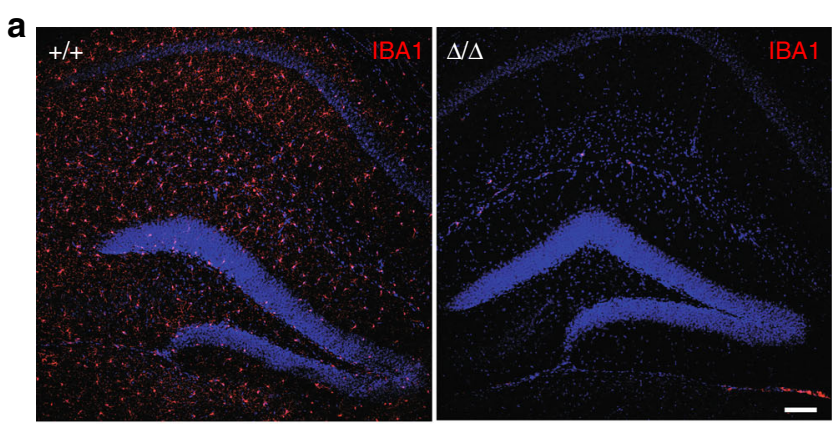

b
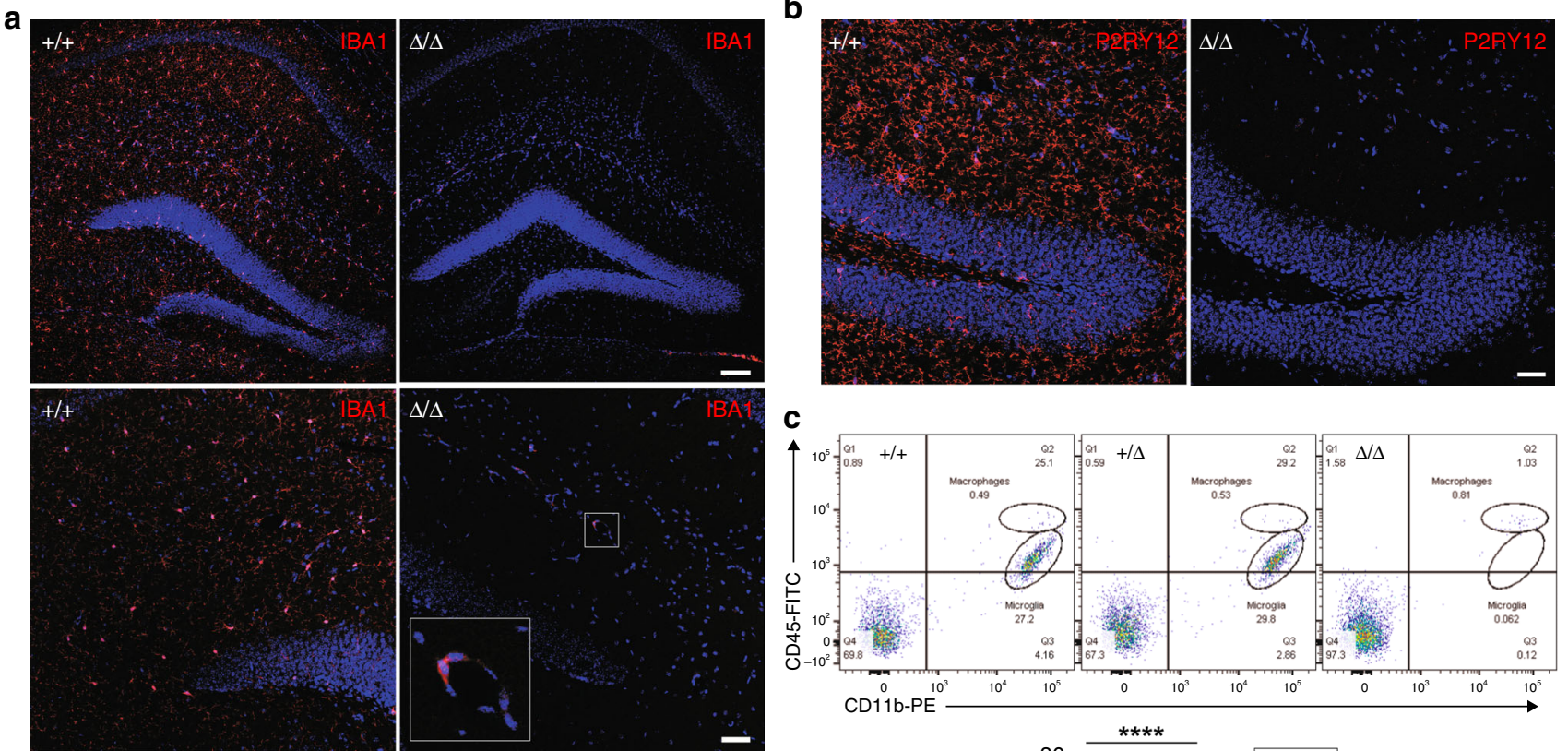

C
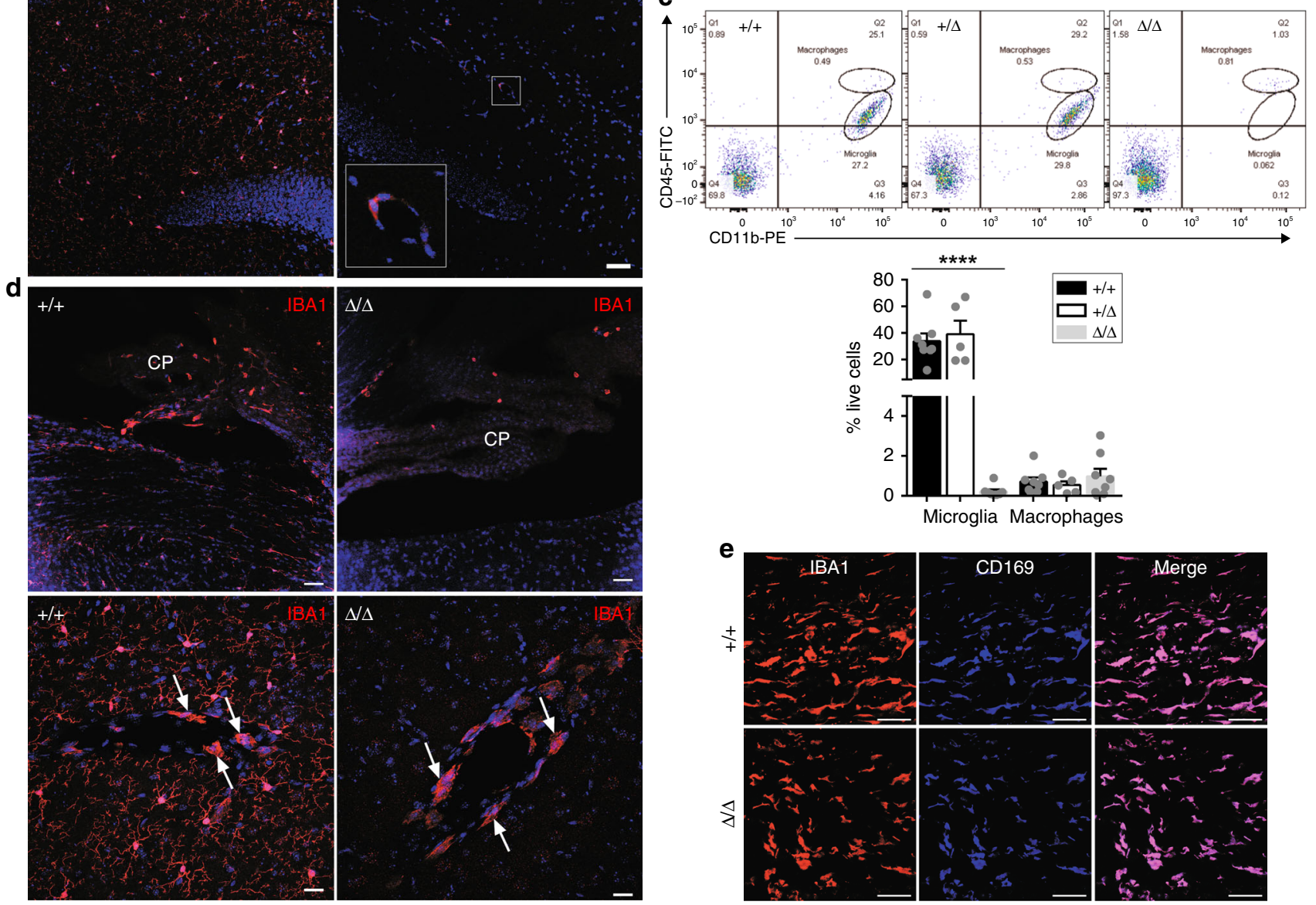

Fig. 7 FIRE deletion results in selective loss of microglia. a Cryosections of adult brains were stained with an antibody against IBA1. Boxed area shows an

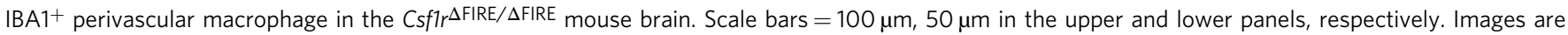
representative of four mice per genotype. $\mathbf{b}$ Cryosections of adult brains were stained with an antibody against P2RY12. Scale bar $=40 \mu \mathrm{m}$. $\mathbf{c}$ Single cell suspensions of myelin-depleted brains from males and females aged 10 weeks to 9 months were analyzed by flow cytometry for CD45 and CD11b

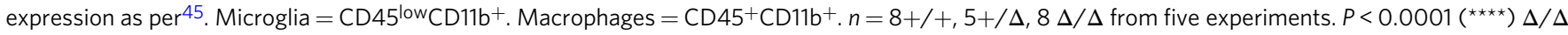
compared to $+/+$. $\mathbf{d}$ Cryosections of adult brains stained with an antibody against IBA1 using the same cohort of mice in $\mathbf{a}$ and $\mathbf{b}$. Upper panel shows the choroid plexus (CP) and white arrows point to perivascular macrophages in the lower panel. Images are representative of three mice. Scale bars $=40$ and $20 \mu \mathrm{m}$ in the upper and lower panels, respectively. e Dissected meningeal dura mater was stained with antibodies against IBA1 and CD169. Images are representative of two mice per genotype. Scale bars $=50 \mu \mathrm{m}$. All source data are provided within a Source Data excel file. Graphs show mean $+\mathrm{SEM}$ and $P$ values were determined by two-tailed $t$-tests

brain and analysis by flow cytometry ${ }^{45}$ confirms the loss of $\mathrm{CD} 45^{\text {low }} / \mathrm{CD} 11 \mathrm{~b}^{+}$microglia which persists up to 9 months of age (Fig. 7c). The full flow cytometry gating strategy is shown in Supplementary Fig. 8a. Heterozygous mutation (haploinsufficiency) of Csfir has been proposed as a model for the human neurodegenerative disease ALSP (adult onset leukoencephalopathy with axonal spheroids and pigmented glia) ${ }^{46}$. However, there is no detectable effect of the heterozygous mutation in mice $\left(C s f 1 r^{\Delta \mathrm{FIRE} /+}\right)$ on microglial numbers. The brain also contains populations of $\mathrm{CD} 45^{\text {hi }}$ classical macrophages associated with the vasculature, meninges, and choroid plexus with a distinct transcriptional profile ${ }^{47,48}$. CD $45^{\text {hi }}$ cells are retained in Csfl $r^{\triangle \text { FIRE/ } \triangle F I R E ~ b r a i n ~ d i g e s t s ~(F i g . ~ 7 c) ~ T h i s ~ w a s ~ c o n f i r m e d ~ b y ~}$ immunolocalization of CD169, shown recently to be a marker alongside CD206 and LYVE1 for a perivascular macrophage population in most tissues ${ }^{49}$ (Fig. $7 \mathrm{~d}$, e). The brain-to-body weight ratios in adult male and female Csfir $\triangle$ FIRE/ $\Delta$ FIRE mice remains unchanged (Supplementary Fig. 8b). 
Microglia are so abundant in the brain that their gene expression profile is readily detected in total mRNA. The complete loss of microglia in $C s f 1 r^{-1-}$ rat brains was associated with commensurate loss of known microglia-associated transcripts in four brain regions ${ }^{5}$. To confirm the loss of microglia we compared the gene expression profiles of $C s f 1 r^{\triangle \mathrm{FIRE} / \Delta \mathrm{FIRE}}$ and $C s f 1 r^{+/+}$hippocampi. The results, including ANOVA analysis of significantly regulated transcripts, are contained within Supplementary Data 2. Overall, 85 genes are significantly downregulated in the $C s f 1 r^{\Delta \mathrm{FIRE} / \Delta \mathrm{FIRE}}$ mouse hippocampus and 77 genes from this group (including Csf1r) have been previously shown to be expressed in human and mouse microglia $45,50,51$. Of the top 35 transcripts found by Elmore and colleagues to be down-regulated in the brains of adult mice treated with a CSF1R kinase inhibitor ${ }^{52}, 26$ are also significantly reduced in the hippocampus of $C s f 1 r^{\Delta \mathrm{FIRE} / \Delta \mathrm{FIRE}}$ mice. The gene set affected by the $C s f 1 r^{\Delta \mathrm{FIRE} / \Delta \mathrm{FIRE}}$ mutation includes a small set of novel genes that may be implicated in microglial biology. Amongst these, the immune checkpoint protein, VISTA (Vsir), was recently localized in both human and mouse and was attributed functions in CNS pathologies ${ }^{53}$. In addition, HVCN1 was previously shown to regulate the production of microglial reactive oxygen species ${ }^{54}$. Consistent with the retention of the, $\mathrm{CD} 45^{\text {hi }} \mathrm{CD} 11 \mathrm{~b}^{+} \mathrm{CD} 169^{+}$brain macrophage population in the Csf1 $r^{\triangle \mathrm{FIRE} / \Delta \mathrm{FIRE}}$ mice (Fig. 7b-e), Ptprc (encoding CD45 antigen) and myeloid markers, such as $\operatorname{Mrc1}(C d 206)$, Siglec1, Siglecf, Cd163, Csf2ra/b/b2, Fcgr2b, Tnfrfsf11a (RANK) that have all been associated with these populations ${ }^{47,48}$ are not significantly affected by the deletion of FIRE (Supplementary Data 2). The complete loss of detectable Csfir expression implies that the perivascular and brain-associated macrophages, like blood monocytes and the macrophages of the BM, have lost Csf1r expression but are nevertheless produced normally.

Microglia have been implicated in oligodendrocyte maturation and synaptic plasticity 55 . The loss or induced depletion of microglia in other models leads to alterations in synaptic pruning, hippocampal neurogenesis, myelination, oligodendrocyte maturation, and astrocyte activation ${ }^{56}$. By contrast to phenotypes reported in $C s f 1^{\text {op/op }}$ and $C s f 1 r^{-1-}$ mice ${ }^{4}$, we observe no evidence of auditory or visual abnormalities in the $C s f 1 r^{\Delta \text { FIRE/ }} \Delta$ FIRE mice. Sensory neuronal deficits have been associated with deficient hippocampal neurogenesis but in the $C s f 1 r^{\Delta \mathrm{FIRE} / \Delta \mathrm{FIRE}}$ mice there is no loss of expression of markers associated with neuronal progenitor cells (such as doublecortin (Dcx), and Sox $2^{57}$ ) (Supplementary Data 2). Nandi et al. ${ }^{58}$ claimed that Csflr is expressed in subsets of neuronal progenitors, and noted the relative loss of excitatory neurons, expressing Cux1 or Ctip2 (BCll1b) in the Csf1r ${ }^{-1-}$ mouse brain. CUX1 mRNA was also reduced in the brain in a recently described human patient with a homozygous CSF1R mutation ${ }^{59}$. Neither transcript is downregulated in the hippocampus of $C s f 1 r^{\Delta \mathrm{FIRE} / \Delta \mathrm{FIRE}}$ mice (Supplementary Data 2).

Zhang et al. ${ }^{60}$ used expression profiles of isolated cells to identify a set of signatures of brain cell populations that can support deconvolution of whole brain expression data. As shown in Supplementary Fig. 8c, there is no significant effect of the $C s f 1 r^{\Delta \mathrm{FIRE} / \Delta \mathrm{FIRE}}$ mutation on any cell-type-specific signature (neuron, astrocyte, oligodendrocyte, endothelium) other than microglia. The lack of impact on astrocyte numbers or morphology was confirmed by immunolocalization of the specific markers GFAP and SOX9 in the hippocampus, as well as the striatum, olfactory bulbs, and cerebellum (Supplementary Fig. 8d). Whereas microglia have been implicated as major sources of trophic factors, mRNA expression of $B d n f$, $\operatorname{Igf1}, \mathrm{Tg} f b 1,2$, and 3 is not affected by the absence of microglia in Csfir $r^{\triangle \text { FIRE/ }}$ FIRE mice (Supplementary Data 2). Similarly, macrophages and microglia selectively express very high levels of lysosomal-associated genes (e.g. Lamp1, Ctsb, Atp6v0e) and receptors for apoptotic cells (e.g. Mertk, Axl) compared to whole brain regions (e.g. see biogps.org) but the loss of microglia has no effect on the expression of any lysosomeassociated transcripts in the hippocampus of $C s f 1 r^{\Delta \mathrm{FIRE} / \Delta \mathrm{FIRE}}$ mice. This conclusion is consistent with the proposal that astrocytes are potentially also engaged with apoptotic cell clearance ${ }^{61}$ and might fill this role in the absence of microglia.

Embryonic macrophage development in $C s f 1 r^{\Delta F I R E / \Delta F I R E ~ m i c e . ~}$ Many tissue resident macrophages are seeded during embryonic development from the yolk sac and fetal liver progenitors ${ }^{24,30,41,44,62,63}$. Csflr ${ }^{-l-}$ mice lack yolk sac-derived macrophages and microglia but develop fetal monocytes ${ }^{44}$. Treatment of a pregnant mouse with anti-CSF1R resulted in the loss of embryonic macrophages derived from the yolk sac, whereas circulating liver-derived monocytes were unaffected ${ }^{63}$. As shown in Fig. 8a the Csf1r $r^{\Delta \text { FIRE/ } \triangle \text { FIRE }}$ embryos develop normally but the overall density of $\mathrm{IBA}^{+}$macrophages throughout the body is greatly reduced. In the critical window in which liver hematopoiesis is established and the blood-brain barrier is closed (embryonic day 12-13), IBA1 ${ }^{+}$ameboid microglia are prevalent in controls but undetectable in Csf1r $1 r^{\triangle \mathrm{FIRE} / \Delta \mathrm{FIRE}}$ embryos (Fig. 8b-d). Numerous macrophages expressing the CSF1R-ECFP and CSF1R-EGFP reporter genes line the ventricular and external surfaces of the developing brain from $9.5 \mathrm{dpc}^{10,64}$. These cells are absent in Csf1r $\Delta$ FIRE/ $\Delta$ FIRE embryos (Fig. 8b, c). Only occasional monocyte-like cells associated with blood vessels are seen in the brain parenchyma (Fig. 8b). By contrast, IBA1 ${ }^{+}$monocytes/ macrophages are detected in the meninges and choroid plexus in both control and mutant embryos (Fig. 8c, d), consistent with our findings in the adult brain (Fig. 7). There is also a reduction in the $\mathrm{IBA}^{+}$macrophages in the fetal liver at the same stages of development (Fig. 8e). We conclude that the $C s f 1 r^{\Delta \text { FIRE/ } / \text { FIRE }}$ mice recapitulate the phenotype of $\mathrm{Csflr}^{-1-}$ mice in early hematopoiesis, producing a selective deficiency of yolk sacderived cells. The major macrophage populations that are retained in the adult $C s f 1 r^{\Delta \mathrm{FIRE} / \Delta \mathrm{FIRE}}$ mice (liver, lung, spleen, intestine, dermis, etc.) are established late in gestation and postnatally from monocytes derived from progenitors in the fetal liver $24,30,44,62,63$. This conclusion is consistent with analysis of expression of tissue macrophage-specific genes across an embryonic gene expression time course ${ }^{65}$.

The chromatin landscape of Csf1r. Chromatin analysis and ChIP-seq data identifies at least four other Csf1r enhancers that are active in mouse BMDM. Each is bound by JUN, STAT1, PU.1, and CEBPA, which also bind cooperatively to many other macrophage-associated enhancers (reviewed in ref. ${ }^{9}$ ). To extend these findings to other macrophage populations, we explored data from a previous study that compared the enhancer profiles of monocytes, microglia, and macrophages isolated from lung, liver, spleen, intestine, and peritoneal cavity ${ }^{66}$. Peaks of H3K4me1 methylation (indicative of poised enhancers) appear throughout the Csf1r locus and extend upstream into the neighboring Pdgfrb gene (Fig. 9a). This extended H3K4mel signal is almost undetectable in neutrophils, which express Csfir mRNA and Csf1r reporter genes at low levels but do not express surface CSF1R ${ }^{11}$. These additional elements lie outside the $3.5 \mathrm{~kb}$ promoter region, and intron containing FIRE, used in production of Csf1r-reporter genes (Fig. 9a). The ATAC-seq data revealed heterogeneity amongst monocyte-macrophage populations. FIRE is clearly a major peak of open chromatin detected in microglia and peritoneal macrophages, consistent with the impact of deletion of this 
a

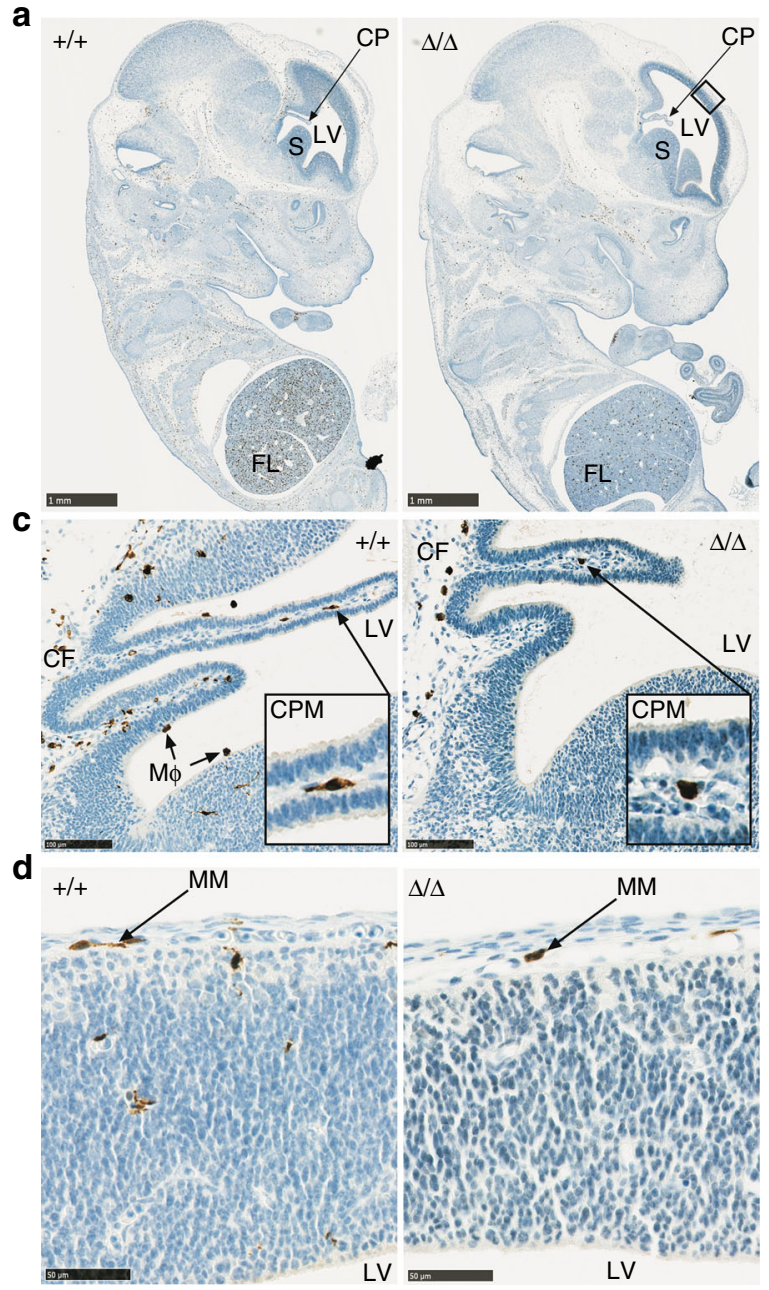

b
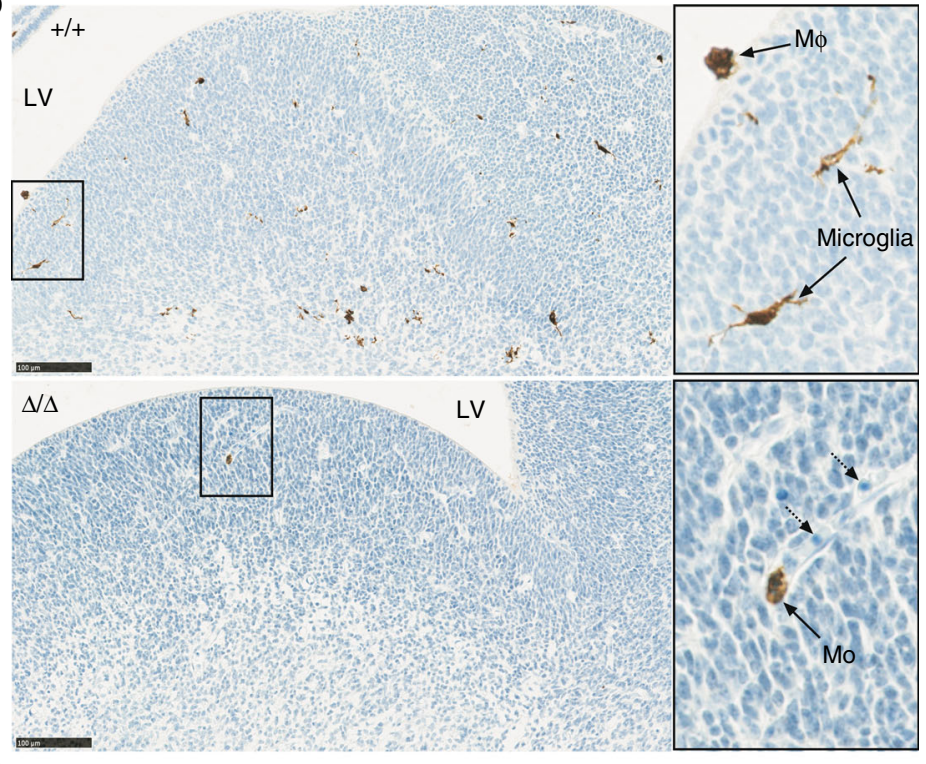

e

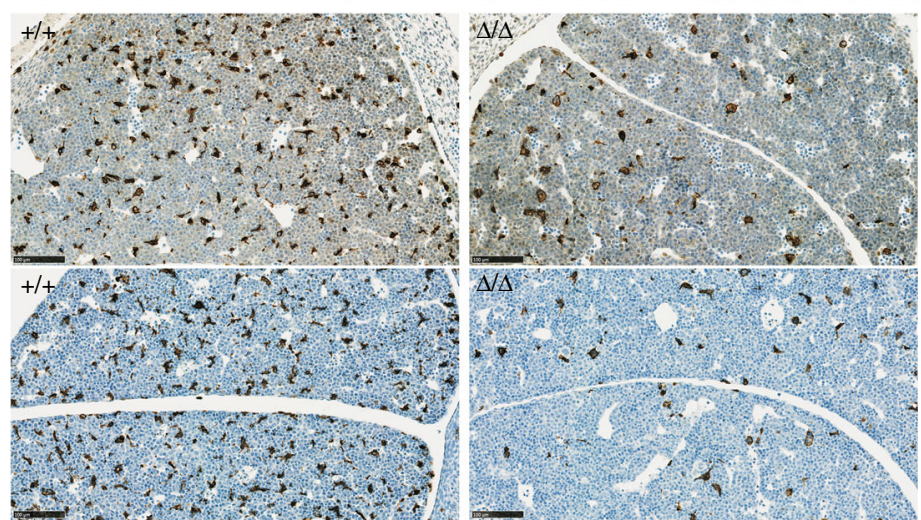

Fig. 8 FIRE deletion results in a reduction of embryonic macrophages. a Formalin-fixed paraffin-embedded embryos (E13) were stained with antibodies against IBA1. Images are representative of seven embryos per genotype at E12, E12.5, and E13 and four repeat experiments. CP choroid plexus, LV lateral ventricle, S striatum, FL fetal liver. Black rectangle indicates the area magnified for panel $\mathbf{d}$. Scale bar $=1 \mathrm{~mm}$. b View of the striatum from E12.5 embryos highlighting IBA1+ microglia and ventricular macrophages $(M \Phi)$ in the control embryo and a monocyte (Mo) in the FIRE deficient embryo. Dotted arrows point to immature red blood cells in a blood vessel. Scale bar $=100 \mu \mathrm{m}$. c Images of the choroid plexus in the lateral ventricle (LV) of E12.5 embryos. CPM

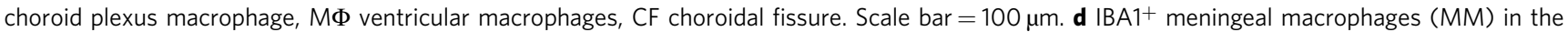
forebrain (boxed area in panel a) of control and Csf1r $\triangle \mathrm{FIRE} / \Delta \mathrm{FIRE}$ embryos at E12.5. Scale bar $=50 \mu \mathrm{m}$. e IBA $1^{+}$fetal liver monocytes at E12 and E13 in upper and lower panels, respectively. Scale bar $=100 \mu \mathrm{m}$

element (Fig. 9b). By contrast, the signal associated with FIRE is barely detectable in isolated lung and intestinal macrophages. Indeed, the promoter-associated ATAC-seq signal is barely detectable, consistent with relatively low expression of Csflr mRNA in these cells $35,67,68$ and lack of phenotype observed in these tissues in Csf1 $1 r^{\Delta \mathrm{FIRE} / \Delta \mathrm{FIRE}}$ mice ${ }^{21}$. The ATAC-seq data in Fig. $9 \mathrm{~b}$ reveals multiple additional elements in cells that clear the majority of CSF1 from the circulation (notably in KC and splenic macrophages), that likely contribute to the redundancy of FIRE and to the high level of Csfir expression in these cell types ${ }^{11}$. One of these elements includes the distal promoter element (DPE). Located $5^{\prime}$ of the macrophage transcription start site, the DPE also has tissue-specific functions ${ }^{64}$. It includes a separate transcription start site cluster used by osteoclasts ${ }^{69}$. Expression of an amplified Csfir-ECFP (MacBlue) reporter transgene, in which the DPE was deleted from the promoter, was undetectable in most tissue macrophages ${ }^{64}$. The exceptions that retained reporter gene expression were those populations impacted in the Csfir $r$ FIRE/ $\triangle \mathrm{FIRE}$ mouse; embryonic macrophages, BM progenitors and monocytes, microglia, Langerhans cells, and peritoneal macrophages. Therefore, we conclude that FIRE and the DPE interact to control expression of Csflr in embryonic macrophages and in some tissue-resident macrophage populations but that both are partly redundant.

\section{Discussion}

We show that the regulatory element, FIRE, is not required to support Csf1r transcription in all macrophage populations. FIRE is essential for the generation or maintenance of specific CSF1Rdependent tissue macrophages, including those of the embryo, the brain (microglia), skin (Langerhans cells), peritoneum, heart, and kidney but redundant for expression of Csf1r mRNA in adult mononuclear phagocyte populations in the intestine and lung. FIRE is not required for the development of CSF1R-dependent macrophages in other locations (i.e. dermis, spleen, liver, and bone). The microglial population of the brain is established early in development from yolk sac-derived progenitors. Apart from a transient postnatal monocyte influx, microglia are maintained by self-renewal in a CSF1R-dependent manner ${ }^{70}$. The loss of $\mathrm{IBA}^{+}$cells in Csfl $r^{\Delta \mathrm{FIRE} / \Delta \mathrm{FIRE}}$ embryos (Fig. 8) indicates that 
a

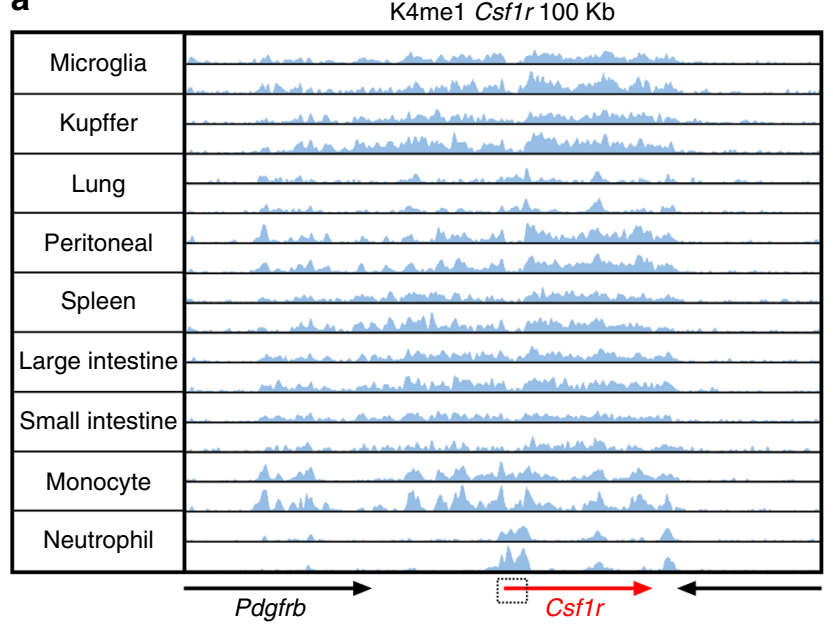

b

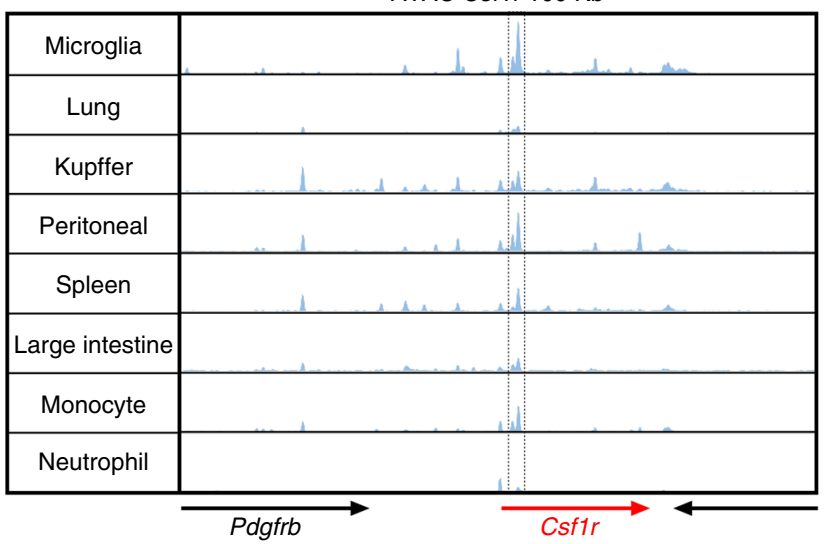

Fig. 9 Chromatin landscape of the mouse Csf1r locus. ChIP-seq and ATACseq data for the $100 \mathrm{~kb}$ window surrounding mouse Csfir for each of the isolated myeloid cell populations shown was extracted from the primary data generated in Lavin et al. ${ }^{66}$. a ChIP-seq data showing the location of poised enhancers (H3K4me1). The boxed area indicates the $3.5 \mathrm{~kb}$ promoter region used in the production of Csf1r reporter genes. b ATACseq data showing the locations of open chromatin. The dotted lines surround the double peak in the second intron; the more prominent peak is FIRE

microglial development fails early in embryogenesis. This is consistent with the apparent failure of macrophage production from $C s f 1 r \Delta \mathrm{FIRE} / \Delta \mathrm{FIRE}$ EB in the E14 ESC model (Fig. 1f).

The relative independence of the large majority of tissueresident macrophages on the enhancer activity of FIRE begs the question of why this element is so highly conserved across evolution. Enhancer redundancy (i.e. the presence of shadow enhancers), is widespread in eukaryotic developmental systems ${ }^{71}$. Nevertheless, redundancy is often incomplete and the nonoverlapping functions presumably explain why multiple enhancers within a locus are conserved across evolution ${ }^{71}$. The core element within FIRE, conserved from reptiles to humans, contains binding sites for AP1 and PU.1 that are essential for enhancer activity ${ }^{14}$. Colocalization of AP1 and PU.1 motifs is a feature of many mouse macrophage-specific constitutive enhancers $^{72}$. The non-redundant role of FIRE in Csflr transcription in only a subset of macrophages may be a consequence of tissuespecific expression of transcription factors that bind selectively to this region of the Csfir locus. Candidate regulators have been identified in many CSF1R-dependent macrophage populations.
For example, microglia are uniquely dependent upon specific transcriptional regulators encoded by Sall $1^{73}$ and $\operatorname{Irf8}{ }^{74}$. Whilst multiple open chromatin sites are bound by PU.1, CEBPA, and other transcription factors in BMDM, IRF8 bound strongly and exclusively to FIRE (reviewed in ref. ${ }^{9}$ ). Irf8 mRNA is coexpressed with Csflr in the earliest committed monocyte progenitors ${ }^{75}$ and upregulated in erythro-myeloid progenitors in parallel with Csflr during early embryonic development ${ }^{67}$. Renal macrophages, which are also lost in Csf1 $r \Delta \mathrm{FIRE} / \Delta \mathrm{FIRE}$ mice, along with blood monocytes, share high gene expression of Irf8 with microglia ${ }^{67}$. RUNX1 is another transcription factor that interacts with FIRE in both mouse and human macrophages and controls CSF1-dependent macrophage growth ${ }^{76}$. Accordingly, Runx1 mRNA is expressed in the earliest progenitors of CSF1R-positive microglia $^{44}$ and is amongst the transcription factors downregulated in the hippocampus in Csfir $\triangle \mathrm{FIRE} / \Delta \mathrm{FIRE}$ mice (Supplementary Data 1).

CSF1R deficiency causes embryonic or perinatal death in most mouse strains and the few surviving Csf1r mutant mice have numerous developmental abnormalities ${ }^{77}$. The Csfir $r^{\Delta \mathrm{FIRE} / \Delta \mathrm{FIRE}}$ mice that are born survive and thrive. Aside from survival, other major differences between the pleiotropic impacts of $C s f 1 r^{\Delta \mathrm{FIRE} / \Delta \mathrm{FIRE}}$ and $C s f 1 r^{-1-}$ mutations ${ }^{4,77}$ are clearly related to the selective retention of most Csf1r-dependent macrophage populations in mice with the FIRE deletion. The expression profiling of the hippocampus supports the view that trophic and phagocytic functions attributed to microglia can be compensated in part by other cells. Notably, neuronal development, including expression of markers such as Cuxl, is unaffected by the complete loss of Csfir expression. This observation argues strongly against a functional role for Csfir in neuronal progenitors ${ }^{58}$ and instead supports that Csf1r is expressed and functional only in microglia ${ }^{19}$. So, we suggest that the reported developmental and homeostatic roles of Csf1r-dependent macrophages, including microglia and those of the epidermis, peritoneum, kidney, and heart are at least partly redundant. By extension, the loss of most peripheral macrophages and the associated systemic impacts of that loss must contribute to the severe brain developmental abnormality observed in $\mathrm{Csf} 1 \mathrm{r}^{-/-}$mice ${ }^{19,78}$. Severe postnatal growth retardation is a shared feature of Csf1 and Csfir mutant mice and rats ${ }^{4,5}$ and is a consequence of impacts on the growth hormone (GH)/insulin-like growth factor 1 (IGF1) axis (reviewed in ref. ${ }^{79}$ ). By contrast to the Csfir-deficient mice, liver macrophages develop normally in $C s f 1 r^{\Delta \mathrm{FIRE} / \Delta \mathrm{FIRE}}$ animals (Fig. $4 \mathrm{a}$ and Supplementary Fig. 4a) and somatic growth, as well as the male-female sexual dimorphism in body weight gain, is unaffected by the mutation. In Csf 1 mutant rats, there was no postnatal surge in circulating IGF1 ${ }^{79}$ ) and the Csflr mutation in rats reduced expression of both $G h r$ and Igf 1 mRNA in the liver ${ }^{5}$. Circulating IGF1 in turn crosses the blood brain barrier and regulates many aspects of neuronal health (reviewed in ref. ${ }^{80}$ ).

In overview, our objective in this study was to investigate the role of a conserved enhancer in a key macrophage-expressed gene. We have confirmed the functional importance of FIRE in specific macrophage populations. In the process, we have generated a unique mouse model in which to identify non-redundant functions of specific resident tissue macrophages, especially those of the brain, heart, skin, kidney, and peritoneum, in mice that are otherwise healthy and fertile.

\section{Methods}

CRISPR design and plasmid preparation. gRNAs were designed to complement the flanking regions of the FIRE element of intron 2 (from 2646 to $3015 \mathrm{bp}$ downstream of the end of exon 2; NCBI Reference Sequence: NM 001037859.2) using the online tool at http://crispr.mit.edu. Table 1 shows the guides designed, the corresponding protospacer-adjacent motif (PAM) and the complementary 
Table 1 CRISPR guide RNAs

\begin{tabular}{lllll} 
Guide & Top BbSI-flanking sequence & Guide sequence & Bottom BbSI-flanking sequence \\
\hline US1-top & CACC & 5' GAGTCCCTCAGTGTGTGAGA & - & PAM \\
US1-bottom & - & 3' TCTCACACACTGAGGGACTC & CAAA \\
DS1-top & CACC & 5' GGGATGACACAACGGTTTCC & - & AGG \\
DS1-bottom & - & 3' GGAAACCGTTGTGCATCCC & CAAA & - \\
DS2-top & CACC & 5' CAATGAGTCTGTACTGGAGC & - & TGG \\
DS2-bottom & - & 3' GCTCCAGTACAGACTCATTG & CAAA & AGG \\
\hline
\end{tabular}

US upstream FIRE, DS downstream FIRE, PAM protospacer-adjacent motif

BbSI-overhang sequences. Top and bottom oligonucleotides corresponding to each gRNA were aligned, phosphorylated, and cloned into the pSpCas9(BB)-2A-GFP (Px458) plasmid (Addgene), and transformed into competent DH5a E. coli. Following verification of gRNAs insertion by Sanger sequencing, plasmids were purified using endotoxin-free MaxiPrep (QIAGEN).

Cell culture. RAW 264.7 macrophages (ATCC ${ }^{\oplus}$ TIB-71 ${ }^{\text {m) }}$ ) were cultured on Sterilin-plastic dishes in RPMI 1640 medium (Sigma) supplemented with $10 \%$ endotoxin-free FBS (GE Healthcare), $2 \mathrm{mM}$ GlutaMAX (Invitrogen), $25 \mathrm{U} / \mathrm{mL}$ of penicillin, and $25 \mu \mathrm{g} / \mathrm{mL}$ of streptomycin (Gibco). Cells were incubated at $37^{\circ} \mathrm{C}$ in a $5 \% \mathrm{CO}_{2}$ humidified incubator. E14 (129P2/OlaHsd) mouse ESC (ATCC ${ }^{\oplus}$ CRL$1821^{\text {min }}$ ) were cultured on porcine gelatin-coated (Sigma) flasks in GMEM (Invitrogen) supplemented with 10\% ESC-tested FBS (Hyclone), 2 mM GlutaMAX (Invitrogen), 1X MEM non-essential amino acids, $1 \mathrm{mM}$ sodium pyruvate, $0.1 \mathrm{mM}$ 2-mercaptoethanol (Gibco), and $100 \mathrm{U} / \mathrm{mL}$ leukemia inhibitory factor (LIF, Merck), at $37^{\circ} \mathrm{C}$ in a $5 \% \mathrm{CO}_{2}$ humidified incubator.

Generation of CRISPR/Cas9 deletions in vitro. E14 cells were passaged twice and plated at $8 \times 10^{4} \mathrm{cells} / \mathrm{cm}^{2}$ for $24 \mathrm{~h}$ prior to transfection. Cells were transfected with a total of $2 \mu \mathrm{g}$ DNA (i.e. pairs of Cas9-2A-EGFP vectors, each expressing single gRNAs: US1+DS1 or DS2) using the Amaxa P3 Primary cell 4DNucleofector X Kit (Lonza) and incubated with supplemented GMEM for $36 \mathrm{~h}$. Cells were then resuspended in Dulbecco's phosphate buffered saline (DPBS, Sigma), diluted in sterile Baxter water (TPS Healthcare/Baxter), supplemented with $2 \% \mathrm{FBS}$ and kept at $4{ }^{\circ} \mathrm{C}$. The $\mathrm{GFP}^{+}$cells were collected with a FACS Aria IIIu cell sorter (BD). To generate single cell clones of CRISPR-modified E14 cells, sorted pools of $\mathrm{GFP}^{+}$cells were plated on $0.1 \%$ gelatin-coated $100 \mathrm{~mm}$ culture dishes at a density of $18 \mathrm{cells} / \mathrm{cm}^{2}$ and single colonies picked approximately 2 weeks later. RAW 264.7 cells $\left(5 \times 10^{6}\right)$ were resuspended in $250 \mu \mathrm{L}$ of culture medium containing $10 \mu \mathrm{L}$ of DPBS $\pm 20 \mu \mathrm{g}$ DNA (as pairs of FIRE-targeting Cas9-2A-EGFP vectors, as specified above) and incubated at RT for $10 \mathrm{~min}$. Electroporation was performed in $0.4 \mathrm{~cm}$ electroporation cuvettes (BioRad) using the BioRad Gene Pulser II (BioRad), at $320 \mathrm{~V}$ and a capacitance of $950 \mu \mathrm{F}$. Cells were cultured for $36 \mathrm{~h}$ and $\mathrm{GFP}^{+}$cells were single-cell sorted into polystyrene flat-bottom 96-well plates, using a FACS Aria IIIu cell sorter (BD).

PCR analysis and sequencing of transfected E14 ESC and RAW 264.7 cells Isolation of DNA from sorted pools of $\mathrm{GFP}^{+}$cells (either E14 ESC or RAW 264.7 cells), or from cells cultured in plates other than 96-well plates was performed with the QIAGEN DNeasy Blood and Tissue Kit (QIAGEN), according to the manufacturer's instructions. Genomic DNA from single-cell-derived clones cultured in 96-well plates was isolated by adding a 1:1 mix of DirectPCR Lysis Reagent (Viagen) and nuclease-free water (Invitrogen) supplemented with $20 \mu \mathrm{g}$ of Proteinase $\mathrm{K}$ (QIAGEN), per well. Cells were incubated in a heated orbital shaker at $200 \mathrm{rpm}$ and $55^{\circ} \mathrm{C}$, for $12 \mathrm{~h}$ and Proteinase $\mathrm{K}$ was inactivated at $85^{\circ} \mathrm{C}$ for $45 \mathrm{~min}$. Conventional PCR was performed using $50 \mathrm{ng}$ DNA per reaction, Taq DNA polymerase (Invitrogen) and the primers F-5': GCTGCCCTGTCACTGTGTA and R-5':

TCGTTTCCCATCCCAGGA, at an annealing temperature of $55^{\circ} \mathrm{C}$. PCR products were run by electrophoresis to obtain a Csf1r $r^{+/+}$of $925 \mathrm{bp}$, or Csfir $\triangle \mathrm{FIRE} / \Delta \mathrm{FIRE}$ amplicons of either $485 \mathrm{bp}$ (CRISPR pair: US1 and DS1) or $507 \mathrm{bp}$ (CRISPR pair: US1 and DS2). PCR amplicons were separated by standard electrophoresis, purified with the QIAquick PCR purification kit (QIAGEN) and then sequenced using the oligonucleotides listed in Supplementary Table 1.

DNA isolation and genotyping. Mouse ear biopsies were digested overnight at $50^{\circ} \mathrm{C}$, in mouse biopsy buffer containing $100 \mathrm{mM}$ Tris- $\mathrm{HCl}$ (Sigma) $\mathrm{pH} 8.5,0.2 \%$ SDS (Ambion), $200 \mathrm{mM} \mathrm{NaCl}$ (Sigma), $5 \mathrm{mM}$ EDTA (Ambion), and $160 \mu \mathrm{g}$ Proteinase K (QIAGEN). Digested tissues were centrifuged at $12,000 \times \mathrm{g}$ for $15 \mathrm{~min}$ at $4{ }^{\circ} \mathrm{C}$ and DNA was precipitated through the isopropanol-ethanol method. DNA pellets were dried at RT, resuspended in nuclease-free water and quantified using a Nanodrop $^{\text {mu }} 1000$ (Thermo Fisher Scientific). PCR amplification was performed as described above and products were purified using the OIAquick PCR purification kit (QIAGEN) and sequenced using the oligonucleotides listed in Supplementary Table 1 .
Phagocytosis assay. RAW 264.7 cells were cultured in high glucose DMEM (Gibco) supplemented with 10\% endotoxin-free FBS (GE Healthcare) and $1 \mathrm{mM}$ GlutaMAX (Life Technologies). Cells were incubated at $37^{\circ} \mathrm{C}$ for $2 \mathrm{~h}$ in the presence of pHrodo ${ }^{\oplus}$ Red E. coli BioParticles ${ }^{\oplus}$ (Thermofisher) at a ratio of $100 \mu \mathrm{g} / 10^{6}$ cells. Negative control samples were incubated on ice. Cells were analyzed using the BD LSR Fortessa flow cytometer. Dead cells were identified and excluded based upon DAPI uptake.

Flow cytometry. Cells were analyzed by flow cytometry using standard procedures. Inhibition of non-specific binding was achieved by incubating the cells for $30 \mathrm{~min}$ on ice in purified anti-CD16/32 $(1 \mu \mathrm{g} / \mathrm{mL}$, BioLegend, 101302) or hybridoma 2.4G2 supernatant. All antibodies are listed in Supplementary Table 2. Where indicated, cells were fixed and permeabilized prior to staining using the Leucoperm $^{\mathrm{TM}}$ kit (BioRad). Blood was processed using the Uti-Lyse reagent (Agilent). Data analysis was performed in FlowJo v10.0 (Tree Star). Gates were determined with isotype controls or FMO for all experiments.

Differentiation of ESC-derived macrophages. E14 ESCs were harvested and plated at a density of $5 \times 10^{6}$ cells per $50 \mathrm{~mm}$ Sterilin-plastic dishes, using culture medium without LIF. After 4 days, cell debris was removed using $70 \mu \mathrm{m}$ cell strainers. Fresh culture medium containing $50 \mathrm{ng} / \mathrm{mL}$ of rhCSF1 (a gift from Chiron, USA) and $10 \mathrm{ng} / \mathrm{mL}$ of mouse recombinant interleukin 3 (IL3, Invitrogen) was added. After 7 days the culture medium was replaced to contain $100 \mathrm{ng} / \mathrm{mL}$ of rhCSF1 for 10 days, without IL3.

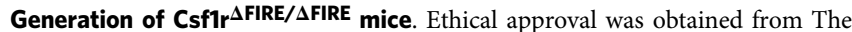
Roslin Institute's and The University of Edinburgh's Protocols and Ethics Committees, under the authority of a UK Home Office Project License under the regulations of the Animals (Scientific Procedures) Act 1986. Mice were bred and housed under specific pathogen-free conditions. $C s f 1 r^{\Delta \mathrm{FIRE} / \Delta \mathrm{FIRE}}$ mice were produced by pronuclear injection of oocytes with the plasmids encoding FIRECRISPRs US1 and DS2 (10 $\mu \mathrm{g}$ of each) and Cas 9 mRNA. Both donor and recipient females were B6CBAF1/J (JAX ${ }^{\mathrm{s}}$, stock number 100011). Founders were then crossed to $\mathrm{C} 57 \mathrm{BL} / 6$ mice and their offspring interbred.

Micro CT. Micro CT was performed on formalin-fixed, paraffin-embedded decalcified femurs. Specimens were held in $1 \%$ agarose in a $20 \mathrm{~mL}$ universal tube before mounting in a Skyscan 1172 desktop micro CT (Bruker). The tubes were then scanned through $360^{\circ}$ using a step of $0.28^{\circ}$ between exposures. A voxel resolution of $6.03 \mu \mathrm{m}$ was obtained using the following control settings: $54 \mathrm{kV}$ source voltage, $185 \mu \mathrm{A}$ source current with an exposure time of $1767 \mathrm{~ms}$. A $0.5 \mathrm{~mm}$ aluminum filter and two frame averaging were used to optimize the scan. After scanning, the data was reconstructed using Skyscan software NRecon v1.6.9 (Bruker). The reconstruction thresholding window was optimized to encapsulate the target image, with the same values used for all bones. Reconstructed bones were oriented around their vertical axis using DataViewer v1.5.2.4 (Bruker). The trabecular region of bone $200 \mu \mathrm{m}$ thick starting $100 \mu \mathrm{m}$ proximal to the primary spongiosa was identified, and volumetric analysis to determine the microarchitecture of this region was performed using CTAn v1.16.4.

Histology and immunohistochemistry. Tissues were fixed in $10 \%$ neutral buffered formalin and processed into paraffin using standard procedures. For examination of tissue architecture, sections were stained with hematoxylin and eosin (H\&E) or Luxol fast blue. Femurs and tails were decalcified in EDTA. Osteoclasts were detected by staining sections with the tartrate-resistant acid phosphatase (TRAP) kit (Sigma), according to instructions.

Goblet cells were stained by treating sections of small intestine with $1 \%$ periodic acid for $7 \mathrm{~min}$ and incubating them in Schiffs reagent for $15 \mathrm{~min}$, with washes in water between steps; followed by counterstaining for $15 \mathrm{~s}$ with Hematoxylin.

For immunohistochemistry, antigen retrieval was performed with Proteinase $\mathrm{K}$ for $5 \mathrm{~min}$ at $37^{\circ} \mathrm{C}$ prior to staining. Tissue-resident macrophages in spleen and liver sections were detected by staining with the rat anti-mouse $\mathrm{F} 4 / 80$ monoclonal antibody (1:600; AbD Serotec, MCA497G). For staining of adult brains, primary antibodies were applied overnight at $4^{\circ} \mathrm{C}$ in a humid chamber. These included 
chicken anti-GFAP (1:500; Cambridge Bioscience, 829401) and rabbit anti-SOX9 (1:500; Millipore, AB5535). Following three washes in PBS, fluorescently conjugated secondary antibodies were applied (1:500; Life Technologies) for $2 \mathrm{~h}$ at $\mathrm{RT}$, then slides were counterstained with DAPI for $10 \mathrm{~min}$ and mounted with Fluoromount G (Cambridge Bioscience). For isolation of epidermal sheets, dissected tail skin was incubated in $2 \mathrm{mg} / \mathrm{mL}$ Dispase II (Sigma) at $37^{\circ} \mathrm{C}$ for $20 \mathrm{~min}$. Epidermal skin samples were fixed overnight in $4 \%$ PFA at $4{ }^{\circ} \mathrm{C}$. After washing with TBS, samples were incubated for $1 \mathrm{~h}$ at RT in permeabilization buffer (PB) - TBS containing $0.25 \%$ gelatin from cold water fish skin (Sigma), $0.5 \%$ Triton X100 (Sigma), and 5\% heat-treated goat serum (Sigma). Epidermal samples were then incubated in PB containing rat anti-mouse MHCII (Clone 2G9, 1:100, BD, $553621)$ and 1:30 goat anti-human Langerin (1:30, Thermo Fisher Scientific, PA547250) overnight at $4{ }^{\circ} \mathrm{C}$. Samples were washed five times for $1 \mathrm{~h}$ at RT in TBS containing $0.02 \%$ Tween 20 (Fisher Scientific) (TBST) followed by incubation overnight at RT with anti-goat and anti-rat Alexa Fluour secondary antibodies (Life Technologies) diluted 1:500 in PB. After washing four times for $1 \mathrm{~h}$ in TBST, epidermal sheets were counterstained with DAPI (Sigma) and mounted in Prolong Gold (Life Technologies).

Frozen sections of $4 \%$ PFA perfused adult brains were processed and stained with rabbit anti-IBA1 (1:500, Wako, 019-19741), rabbit anti-P2RY12 (Sigma, 1:125, HPA013796), and Alexa Fluor ${ }^{\circledR}$ conjugated secondary antibodies using standard procedures.

Dura mater were removed into ice-cold PBS, fixed for $1 \mathrm{~h}$ in $4 \%$ PFA, treated for $30 \mathrm{~min}$ at $37^{\circ} \mathrm{C}$ in PBS containing $20 \mathrm{mM}$ EDTA and then blocked in $3 \% \mathrm{BSA}$, $0.3 \%$ Triton X100 in PBS for 30 min. Whole mount staining steps were performed in blocking buffer containing rabbit anti-IBAl overnight (1:1000, Wako, 01919741), goat anti-rabbit Alexa Fluor $5941 \mathrm{~h}$ (1:400, Life Technologies, A-11012), Alexa Fluor ${ }^{\circ} 647$ anti-mouse CD169 $1 \mathrm{~h}$ (Clone Siglec-1, 1:200, BioLegend, 142408), with washes in buffer in between incubations. Stained tissues were then mounted onto glass slides with mounting medium and cover-slipped. Slides were imaged via a LSM710 confocal microscope (Zeiss) using Zen software.

Image acquisition and quantification. Whole-slide bright field images were acquired using the NanoZoomer slide scanner. Image analysis was performed with the NDP.view software v2.4.26 (Hamamatsu) and with ImageJ v1.46h. For spleen and liver, 10 regions of interest (ROI) were exported per sample as jpg format from the NDP.view files. Signal was quantified from the whole area corresponding to each ROI (i.e. each jpg file). For analysis of small intestine, individual villi were set as a ROI in the jpg file derived from PAS-staining. Villus perimeter, as well as the area and the number of Goblet cells (PAS ${ }^{+}$cells) were quantified. Measurement of villus length was performed in H\&E images where 30 villi were measured per sample, from the mucosal base adjacent to the crypts to the apex, using the "ruler" function of the NDP.view v2.4.26 software. Entire brain tissue sections were imaged using a Zeiss AxioScan SlideScanner and visualized with Zen2 software. Epidermal tail sheets were imaged with a Zeiss LSM 710 confocal microscope and ZEN software. For astrocyte analysis the images were obtained by z-stacks using a Zeiss Axio Scan.Z1 (10x objective) and an Olympus 3i Spinning Disk confocal microscope (30x silicone objective) using SlideBook software. For astrocytes counts, tiff files from max projections were imported to Image J, and the images were converted to 8-bit fluorescence RGB tiff and thresholded. For nuclear counts, watershed segmentation on binary images was used to separate objects and then images were analyzed. Two fields of view were quantified and then the mean was calculated and converted to $\mathrm{mm}^{2}$. To count the proportion of GFAP-positive cells, the number of $\mathrm{GFAP}^{+}$cells was divided by the number of $\mathrm{SOX9}^{+}$nuclei.

Blood analysis. Blood was isolated through cardiac puncture, using syringe and needle coated with $2 \%$ EDTA. Blood was collected into $2 \mathrm{~mL}$ EDTA tubes (BD Vacutainer $^{\circ}$ ) containing $1.8 \mathrm{mg}$ of EDTA per $\mathrm{mL}$ of blood. Total blood cell counts were performed using the ABX Pentra 60 hematology analyzer and differential counts of WBC subsets were performed on blood smears.

BM isolation and cell culture. BM was isolated by flushing femurs with RPMI containing $5 \mathrm{mM}$ EDTA. For flow cytometry analysis, erythrocytes were not lysed to prevent cleavage of CSF1R. For macrophage differentiation, erythrocytes were lysed with RBC lysis buffer (BioLegend) and BM cells were cultured on Sterilinplastic dishes in the presence of rhCSF1 (at $100 \mathrm{ng} / \mathrm{mL}$, a gift from Chiron) for 7 days. Fresh media containing rhCSF1 was added on Day 4.

Isolation of peritoneal cells. Cells for flow cytometry analysis were isolated from mice by lavage of the peritoneal cavity with PBS. Cells were centrifuged at $400 \times g$ for $5 \mathrm{~min}$ at $4{ }^{\circ} \mathrm{C}$ prior to resuspension in PBS containing $2 \%$ endotoxin-free FBS (GE Healthcare).

Preparation of organs for flow cytometry. The spleen, liver, lung, heart, and kidneys were prepared for flow cytometry from non-perfused mice. Organs were removed, chopped finely, and digested in RPMI containing $0.625 \mathrm{mg} / \mathrm{mL}$ collagenase $\mathrm{D}$ (Roche), $0.85 \mathrm{mg} / \mathrm{mL}$ collagenase $\mathrm{V}$ (Sigma), $1 \mathrm{mg} / \mathrm{mL}$ dispase (Life Technologies), and $30 \mathrm{U} / \mathrm{mL}$ DNase (Roche) for $22 \mathrm{~min}$ (lung) or $45 \mathrm{~min}$ (other organs) in a shaking incubator at $37^{\circ} \mathrm{C}$. Organ preparations were passed through
$100 \mu \mathrm{m}$ cell strainers and centrifuged at $300 \times \mathrm{g}$ for $5 \mathrm{~min}$ at $4{ }^{\circ} \mathrm{C}$ prior to resuspension in PBS containing $2 \%$ endotoxin-free FBS. Erythrocytes were then lysed using RBC lysis buffer (BioLegend).

Isolation of intestinal cells. Single cell suspensions of small and large intestines (colon) from non-perfused mice were prepared for flow cytometry. Intestines were opened longitudinally and cut into $2-3 \mathrm{~cm}$ segments in ice-cold PBS and intestinal contents removed by gentle shaking. Tissue segments were incubated at $37^{\circ} \mathrm{C}$ for 20 min whilst shaking in RPMI containing 3\% endotoxin-free FBS (GE Healthcare), $20 \mathrm{mM}$ HEPES (Gibco), $5 \mathrm{mM}$ EDTA (Sigma), $1 \mathrm{mM}$ DTT (Promega), and $100 \mathrm{U} / \mathrm{mL}$ polymyxin B (Sigma). Segments were transferred to RPMI containing $2 \mathrm{mM}$ EDTA and $20 \mathrm{mM}$ HEPES and shaken by hand to ensure optimal dissociation of epithelial cells and lamina propria leukocytes. Tissues were minced with scissors and digested at $37^{\circ} \mathrm{C}$ for $30 \mathrm{~min}$, whilst shaking in RPMI containing $20 \mathrm{mM}$ HEPES, $0.425 \mathrm{mg} / \mathrm{mL}$ Collagenase V (Sigma), $0.625 \mathrm{mg} / \mathrm{mL}$ Collagenase D (Roche), $1 \mathrm{mg} / \mathrm{mL}$ Dispase (Gibco), and $30 \mu \mathrm{g} / \mathrm{mL}$ DNase (Roche). Cell suspen sions were passed through $70 \mu \mathrm{m}$ then $40 \mu \mathrm{m}$ cell strainers in RPMI containing $10 \%$ endotoxin-free FBS and $100 \mathrm{U} / \mathrm{mL}$ polymyxin B. After centrifugation at $400 \times g$ at $4{ }^{\circ} \mathrm{C}$ for $5 \mathrm{~min}$, including a wash step, cells were resuspended in PBS containing $2 \%$ FBS for flow cytometry.

Myelin depletion of brains and isolation of cells. Myelin-depleted brain suspensions were prepared from saline perfused mice for flow cytometry. Whole brains were finely minced with scissors in ice-cold Hank's balanced salt solution (HBSS, Sigma, without calcium or magnesium) and centrifuged at $400 \times g$ for $5 \mathrm{~min}$ at $4{ }^{\circ} \mathrm{C}$. Minced brains were then digested in HBSS containing $50 \mathrm{U} / \mathrm{mL}$ Collagenase IV (Gibco), $100 \mu \mathrm{g} / \mathrm{mL} \mathrm{Na-Tosyl-L-lysine} \mathrm{chloromethyl} \mathrm{ketone} \mathrm{hydro-}$ chloride (Sigma), $5 \mathrm{U} / \mathrm{mL}$ DNase I (Roche) and $8.5 \mathrm{U} / \mathrm{mL}$ Dispase (Gibco) for $1 \mathrm{~h}$ at $37^{\circ} \mathrm{C}$ whilst shaking. The digested tissue was homogenized in a glass Dounce tissue grinder for 20 passes and an equal volume of HBSS containing $10 \%$ endotoxin-free FBS was then added. After centrifugation at $400 \times g$ for $5 \mathrm{~min}$ at $4{ }^{\circ} \mathrm{C}$ the cell pellet was resuspended in $16 \mathrm{~mL} 35 \%$ Percoll in HBSS, overlaid with $10 \mathrm{~mL}$ HBSS and incubated on ice for $5 \mathrm{~min}$. The gradient was centrifuged at $800 \times g$ for $45 \mathrm{~min}$ at $4{ }^{\circ} \mathrm{C}$ with no brake. The cell pellets were then resuspended in PBS containing $2 \%$ FBS for flow cytometry.

RNA isolation and microarrays. For RNA isolation, tissues from saline perfused mice were snap frozen, and subsequently disrupted in the Precellys24 Homogenizer (Bertin Instruments). RNA isolation was performed using the RNeasy Plus Mini kit (QIAGEN). Library preparation and hybridization to the Affymetrix Mouse Gene 1.0 ST array was performed by Edinburgh Genomics, University of Edinburgh. CEL files were RMA normalized and annotated in R/Bioconductor. Analysis of differential expression was performed using the Affymetrix Transcription Analysis Console (Thermo Fisher).

Analysis of hippocampi microarray data. These data were interrogated with sets of genes whose expression is highly enriched in specific cell types of the brain (astrocytes, oligodendrocytes, microglia, neurons, brain endothelial cells). These sets were curated from published RNA-seq data (GEO, accession number GSE73721) describing the transcriptome of astrocytes, oligodendrocytes, microglia, neurons, and brain endothelial cells ${ }^{60}$. The inclusion criteria consisted of (a) being expressed at least five-fold higher in the cell type of interest than all other cell types and (b) being expressed at least 50 FPKM to enable robust detection in the mixed cell type environment of a whole hippocampus.

cDNA synthesis and quantitative real-time PCR. Complementary DNA (cDNA) was synthesized from RNA using the SuperScript III. First-Strand Synthesis System and remaining template was removed by incubation with RNase $\mathrm{H}$ (Invitrogen). Oligonucleotides were designed in Primer3 v4.0.0 (available at http://bioinfo.ut.ee/ primer3/). Real-time qPCR reactions were performed using the Fast SYBR green master mix and the 7500 Fast System (Applied Biosystems). Analysis was performed with 7500 software v2.0.6 (Applied Biosystems, Life Technologies Corporation). For analysis of relative changes in gene expression, data were normalized according to the $2^{-\Delta \Delta C T}$ method. Hypoxanthine guanine phosphoribosyl transferase (Hprt) was used as a loading control/housekeeping gene. Oligonucleotide sequences are listed in Supplementary Table 3.

Reporting summary. Further information on research design is available in the Nature Research Reporting Summary linked to this article.

\section{Data availability}

The authors declare that all data supporting the findings in this study are available within the article and its Supplementary Information files or from the corresponding authors on reasonable request. The RNA expression datasets are publicly available in the gene expression omnibus (GEO; https://www.ncbi.nlm.nih.gov/gds) via the accession number GSE108207. Published ATAC-seq and ChIP-seq data used in this manuscript is available via GEO accession number GSE63341. The RNA-seq data used to curate cell type specific 
genes in the brain is available via GEO accession number GSE73721. The data used to identify microglia-enriched genes versus macrophage genes is available via GEO accession number GSE48579. All other data contained within this manuscript is located in the Source Data excel file.

Received: 29 January 2019 Accepted: 15 June 2019

Published online: 19 July 2019

\section{References}

1. Sherr, C. J. et al. The c-fms proto-oncogene product is related to the receptor for the mononuclear phagocyte growth factor, CSF-1. Cell 41, 665-676 (1985).

2. Garceau, V. et al. Pivotal advance: avian colony-stimulating factor 1 (CSF-1), interleukin-34 (IL-34), and CSF-1 receptor genes and gene products. J. Leukoc. Biol. 87, 753-764 (2010).

3. Wang, T. et al. Identification of IL-34 in teleost fish: differential expression of rainbow trout IL-34, MCSF1 and MCSF2, ligands of the MCSF receptor. Mol. Immunol. 53, 398-409 (2013).

4. Dai, X. M. et al. Targeted disruption of the mouse colony-stimulating factor 1 receptor gene results in osteopetrosis, mononuclear phagocyte deficiency, increased primitive progenitor cell frequencies, and reproductive defects. Blood 99, 111-120 (2002).

5. Pridans, C. et al. Pleiotropic impacts of macrophage and microglial deficiency on development in rats with targeted mutation of the Csf1r locus. J. Immunol. 201, 2683-2699 (2018)

6. MacDonald, K. P. et al. An antibody against the colony-stimulating factor 1 receptor depletes the resident subset of monocytes and tissue- and tumorassociated macrophages but does not inhibit inflammation. Blood 116, 3955-3963 (2010).

7. Elmore, M. R. et al. Colony-stimulating factor 1 receptor signaling is necessary for microglia viability, unmasking a microglia progenitor cell in the adult brain. Neuron 82, 380-397 (2014).

8. Rademakers, R. et al. Mutations in the colony stimulating factor 1 receptor (CSF1R) gene cause hereditary diffuse leukoencephalopathy with spheroids. Nat. Genet. 44, 200-205 (2012).

9. Rojo, R., Pridans, C., Langlais, D. \& Hume, D. A. Transcriptional mechanisms that control expression of the macrophage colony-stimulating factor receptor locus. Clin. Sci. 131, 2161-2182 (2017).

10. Sasmono, R. T. et al. A macrophage colony-stimulating factor receptor-green fluorescent protein transgene is expressed throughout the mononuclear phagocyte system of the mouse. Blood 101, 1155-1163 (2003).

11. Hawley, C. A. et al. Csf1r-mApple transgene expression and ligand binding in vivo reveal dynamics of CSF1R expression within the mononuclear phagocyte system. J. Immunol. 200, 2209-2223 (2018).

12. Schulz, C. et al. A lineage of myeloid cells independent of Myb and hematopoietic stem cells. Science 336, 86-90 (2012).

13. Hume, D. A., Wollscheid-Lengeling, E., Rojo, R. \& Pridans, C. The evolution of the macrophage-specific enhancer (Fms intronic regulatory element) within the CSF1R locus of vertebrates. Sci. Rep. 7, 17115 (2017).

14. Sauter, K. A. et al. The function of the conserved regulatory element within the second intron of the mammalian Csf1r locus. PLoS One 8, e54935 (2013).

15. Balic, A. et al. Visualisation of chicken macrophages using transgenic reporter genes: insights into the development of the avian macrophage lineage. Development 141, 3255-3265 (2014).

16. Rosenbauer, F. et al. Acute myeloid leukemia induced by graded reduction of a lineage-specific transcription factor, PU.1. Nat. Genet. 36, 624-630 (2004).

17. Thomas, G. D. et al. Deleting an Nr4al super-enhancer subdomain ablates Ly6C(low) monocytes while preserving macrophage gene function. Immunity 45, 975-987 (2016).

18. Evans, M. J. \& Kaufman, M. H. Establishment in culture of pluripotential cells from mouse embryos. Nature 292, 154-156 (1981)

19. Erblich, B., Zhu, L., Etgen, A. M., Dobrenis, K. \& Pollard, J. W. Absence of colony stimulation factor-1 receptor results in loss of microglia, disrupted brain development and olfactory deficits. PLoS One 6, e26317 (2011).

20. Akcora, D. et al. The CSF-1 receptor fashions the intestinal stem cell niche Stem Cell Res. 10, 203-212 (2013).

21. Sehgal, A. et al. The role of CSF1R-dependent macrophages in control of the intestinal stem-cell niche. Nat. Commun. 9, 1272 (2018).

22. Pollard, J. W., Hunt, J. S., Wiktor-Jedrzejczak, W. \& Stanley, E. R. A pregnancy defect in the osteopetrotic (op/op) mouse demonstrates the requirement for CSF-1 in female fertility. Dev. Biol. 148, 273-283 (1991).

23. Cohen, P. E., Hardy, M. P. \& Pollard, J. W. Colony-stimulating factor-1 plays a major role in the development of reproductive function in male mice. Mol. Endocrinol. 11, 1636-1650 (1997).

24. Yona, S. et al. Fate mapping reveals origins and dynamics of monocytes and tissue macrophages under homeostasis. Immunity 38, 79-91 (2013).
25. Kaur, S. et al. Role of bone marrow macrophages in controlling homeostasis and repair in bone and bone marrow niches. Semin. Cell Dev. Biol. 61, 12-21 (2017).

26. Hettinger, J. et al. Origin of monocytes and macrophages in a committed progenitor. Nat. Immunol. 14, 821-830 (2013).

27. Gow, D. J. et al. Characterisation of a novel Fc conjugate of macrophage colony-stimulating factor. Mol. Ther. 22, 1580-1592 (2014).

28. Witmer-Pack, M. D. et al. Identification of macrophages and dendritic cells in the osteopetrotic (op/op) mouse. J. Cell Sci. 104, 1021-1029 (1993).

29. Lieschke, G. J. et al. Mice lacking both macrophage- and granulocytemacrophage colony-stimulating factor have macrophages and coexistent osteopetrosis and severe lung disease. Blood 84, 27-35 (1994).

30. Tan, S. Y. \& Krasnow, M. A. Developmental origin of lung macrophage diversity. Development 143, 1318-1327 (2016).

31. Shibata, Y., Zsengeller, Z., Otake, K., Palaniyar, N. \& Trapnell, B. C. Alveolar macrophage deficiency in osteopetrotic mice deficient in macrophage colonystimulating factor is spontaneously corrected with age and associated with matrix metalloproteinase expression and emphysema. Blood 98, 2845-2852 (2001).

32. Bain, C. C. et al. Constant replenishment from circulating monocytes maintains the macrophage pool in the intestine of adult mice. Nat. Immunol. 15, 929-937 (2014).

33. Da Silva, C., Wagner, C., Bonnardel, J., Gorvel, J. P. \& Lelouard, H. The Peyer's patch mononuclear phagocyte system at steady state and during infection. Front. Immunol. 8, 1254 (2017).

34. Shaw, T. N. et al. Tissue-resident macrophages in the intestine are long lived and defined by Tim-4 and CD4 expression. J. Exp. Med. 215, 1507-1518 (2018).

35. De Schepper, S. et al. Self-maintaining gut macrophages are essential for intestinal homeostasis. Cell 175, 400-415 e413 (2018).

36. Bain, C. C. et al. Long-lived self-renewing bone marrow-derived macrophages displace embryo-derived cells to inhabit adult serous cavities. Nat. Commun. 7, ncomms11852 (2016)

37. Alikhan, M. A. et al. Colony-stimulating factor-1 promotes kidney growth and repair via alteration of macrophage responses. Am. J. Pathol. 179, 1243-1256 (2011).

38. Stamatiades, E. G. et al. Immune monitoring of trans-endothelial transport by kidney-resident macrophages. Cell 166, 991-1003 (2016).

39. Lavine, K. J. et al. Distinct macrophage lineages contribute to disparate patterns of cardiac recovery and remodeling in the neonatal and adult heart. Proc. Natl Acad. Sci. USA 111, 16029-16034 (2014).

40. Fujiu, K. et al. A heart-brain-kidney network controls adaptation to cardiac stress through tissue macrophage activation. Nat. Med. 23, 611-622 (2017).

41. Dick, S. A. et al. Self-renewing resident cardiac macrophages limit adverse remodeling following myocardial infarction. Nat. Immunol. 20, 29-39 (2019).

42. Wang, Y. et al. IL-34 is a tissue-restricted ligand of CSF1R required for the development of Langerhans cells and microglia. Nat. Immunol. 13, 753-760 (2012).

43. Baranska, A. et al. Unveiling skin macrophage dynamics explains both tattoo persistence and strenuous removal. J. Exp. Med. 215, 1115-1133 (2018).

44. Ginhoux, F. et al. Fate mapping analysis reveals that adult microglia derive from primitive macrophages. Science 330, 841-845 (2010).

45. Grabert, K. et al. Microglial brain region-dependent diversity and selective regional sensitivities to aging. Nat. Neurosci. 19, 504-516 (2016).

46. Chitu, V. et al. Phenotypic characterization of a Csf1 $\mathrm{r}$ haploinsufficient mouse model of adult-onset leukodystrophy with axonal spheroids and pigmented glia (ALSP). Neurobiol. Dis. 74C, 219-228 (2014).

47. Goldmann, T. et al. Origin, fate and dynamics of macrophages at central nervous system interfaces. Nat. Immunol. 17, 797-805 (2016).

48. Van Hove, H. et al. A single-cell atlas of mouse brain macrophages reveals unique transcriptional identities shaped by ontogeny and tissue environment. Nat. Neurosci. 22, 1021-1035 (2019).

49. Chakarov, S. et al. Two distinct interstitial macrophage populations coexist across tissues in specific subtissular niches. Science 363, https://doi.org/ 10.1126/science.aau0964 (2019).

50. Galatro, T. F. et al. Transcriptomic analysis of purified human cortical microglia reveals age-associated changes. Nat. Neurosci. 20, 1162-1171 (2017).

51. Gosselin, D. et al. An environment-dependent transcriptional network specifies human microglia identity. Science 356, https://doi.org/10.1126/ science.aal3222 (2017)

52. Elmore, M. R., Lee, R. J., West, B. L. \& Green, K. N. Characterizing newly repopulated microglia in the adult mouse: impacts on animal behavior, cell morphology, and neuroinflammation. PLoS One 10, e0122912 (2015).

53. Borggrewe, $M$. et al. VISTA expression by microglia decreases during inflammation and is differentially regulated in CNS diseases. Glia 66, 2645-2658 (2018)

54. Kawai, T. et al. Unconventional role of voltage-gated proton channels (VSOP/ Hv1) in regulation of microglial ROS production. J. Neurochem. 142, 686-699 (2017). 
55. Parkhurst, C. N. et al. Microglia promote learning-dependent synapse formation through brain-derived neurotrophic factor. Cell 155, 1596-1609 (2013).

56. Prinz, M., Erny, D. \& Hagemeyer, N. Ontogeny and homeostasis of CNS myeloid cells. Nat. Immunol. 18, 385-392 (2017).

57. Amador-Arjona, A. et al. SOX2 primes the epigenetic landscape in neural precursors enabling proper gene activation during hippocampal neurogenesis. Proc. Natl Acad. Sci. USA 112, E1936-1945 (2015).

58. Nandi, S. et al. The CSF-1 receptor ligands IL-34 and CSF-1 exhibit distinct developmental brain expression patterns and regulate neural progenitor cell maintenance and maturation. Dev. Biol. 367, 100-13 (2012).

59. Oosterhof, N. et al. Homozygous mutations in CSF1R cause a pediatric-onset leukoencephalopathy and can result in congenital absence of microglia. Am. J. Hum. Genet. 104, 936-947 (2019).

60. Zhang, Y. et al. Purification and characterization of progenitor and mature human astrocytes reveals transcriptional and functional differences with mouse. Neuron 89, 37-53 (2016).

61. Cahoy, J. D. et al. A transcriptome database for astrocytes, neurons, and oligodendrocytes: a new resource for understanding brain development and function. J. Neurosci. 28, 264-278 (2008)

62. Hoeffel, G. \& Ginhoux, F. Fetal monocytes and the origins of tissue-resident macrophages. Cell. Immunol. 330, 5-15 (2018).

63. Hoeffel, G. et al. C-Myb(+) erythro-myeloid progenitor-derived fetal monocytes give rise to adult tissue-resident macrophages. Immunity $\mathbf{4 2}$ 665-678 (2015).

64. Sauter, K. A. et al. The MacBlue binary transgene (csflr-gal4VP16/UASECFP) provides a novel marker for visualisation of subsets of monocytes, macrophages and dendritic cells and responsiveness to CSF1 administration. PLoS One 9, e105429 (2014).

65. Summers, K. M. \& Hume, D. A. Identification of the macrophage-specific promoter signature in FANTOM5 mouse embryo developmental time course data. J. Leukoc. Biol. 102, 1081-1092 (2017).

66. Lavin, Y. et al. Tissue-resident macrophage enhancer landscapes are shaped by the local microenvironment. Cell 159, 1312-1326 (2014).

67. Mass, E. et al. Specification of tissue-resident macrophages during organogenesis. Science 353, https://doi.org/10.1126/science.aaf4238 (2016).

68. Schridde, A. et al. Tissue-specific differentiation of colonic macrophages requires TGFbeta receptor-mediated signaling. Mucosal Immunol. 10, 1387-1399 (2017).

69. Ovchinnikov, D. A., DeBats, C. E., Sester, D. P., Sweet, M. J. \& Hume, D. A. A conserved distal segment of the mouse CSF-1 receptor promoter is required for maximal expression of a reporter gene in macrophages and osteoclasts of transgenic mice. J. Leukoc. Biol. 87, 815-822 (2010).

70. Askew, K. et al. Coupled proliferation and apoptosis maintain the rapid turnover of microglia in the adult brain. Cell Rep. 18, 391-405 (2017).

71. Cannavo, E. et al. Shadow enhancers are pervasive features of developmental regulatory networks. Curr. Biol. 26, 38-51 (2016).

72. Ghisletti, S. et al. Identification and characterization of enhancers controlling the inflammatory gene expression program in macrophages. Immunity 32, 317-328 (2010).

73. Buttgereit, A. et al. Sall1 is a transcriptional regulator defining microglia identity and function. Nat. Immunol. 17, 1397-1406 (2016).

74. Hagemeyer, N. et al. Transcriptome-based profiling of yolk sac-derived macrophages reveals a role for Irf8 in macrophage maturation. EMBO J. 35, 1730-1744 (2016).

75. Paul, F. et al. Transcriptional heterogeneity and lineage commitment in myeloid progenitors. Cell 164, 325 (2016).

76. Himes, S. R., Cronau, S., Mulford, C. \& Hume, D. A. The Runx1 transcription factor controls CSF-1-dependent and -independent growth and survival of macrophages. Oncogene 24, 5278-5286 (2005).

77. Chitu, V. \& Stanley, E. R. Regulation of embryonic and postnatal development by the CSF-1 receptor. Curr. Top. Dev. Biol. 123, 229-275 (2017).
78. Bennett, F. C. et al. A combination of ontogeny and CNS environment establishes microglial identity. Neuron 98, 1170-1183 e1178 (2018).

79. Gow, D. J., Sester, D. P. \& Hume, D. A. CSF-1, IGF-1, and the control of postnatal growth and development. J. Leukoc. Biol. 88, 475-481 (2010).

80. Gray, S. M. \& Thorner, M. O. Spatiotemporal regulation of insulin-like growth factor- 1 and its receptor in the brain: is there a role for growth hormone? Endocrinology 158, 229-232 (2017).

\section{Acknowledgements}

This work was supported by the medical research council (MRC) UK grant MR/ M019969/1. R.R. was supported by a doctoral scholarship (application number: 314413, file number: 218819) granted by CONACyT Nuevo Leon-I2T2, Mexico. J.A. was supported by Wellcome Trust grant 201531/Z/16/Z. D.A.H. and K.M.S. are supported by The Mater Foundation. P.H. was supported by the biotechnology and biological sciences research council (BBSRC) grant BB/P013732/1. D.D.O. was supported by MRC grant MR/M010341/1. C.P. is supported by the Simons Foundation Autism Research Initiative Flow cytometry data was generated with support from the Queen's Medical Research Institute (QMRI) and Roslin Institute Flow Cytometry and cell sorting facility, University of Edinburgh. Histology data was generated with support from Easter Bush Pathology and Shared University Research Facilities at the University of Edinburgh. We thank the assistance provided by animal technicians at the University of Edinburgh, particularly Christine Marshall.

\section{Author contributions}

D.A.H. and C.P. conceived the study. P.H. and D.D.O. supervised the CRISPR/Cas9 work R.R., D.A.H. and C.P. wrote the manuscript. K.G., E.W., V.E.M., D.A.D.M, J.P., N.A.M and K.M.S. edited the manuscript. R.R., A.R., D.D.O., L.L., K.G., E.W., M.C., I.G., A.S., Z.M.L., J.A., B.B., I.M., H.D., R.J.L., D.A.D.M., J.D.G., R.W., S.J.J., M.B. and C.P. performed experiments. E.D. and I.A. provided ChIP-seq and ATAC-seq data. R.R., G.E.H., K.M.S., D.A.H. and C.P. analyzed data.

\section{Additional information}

Supplementary Information accompanies this paper at https://doi.org/10.1038/s41467019-11053-8.

Competing interests: The authors declare no competing interests.

Reprints and permission information is available online at http://npg.nature.com/ reprintsandpermissions/

Peer review information: Nature Communications thanks the anonymous reviewer(s) for their contribution to the peer review of this work. Peer reviewer reports are available.

Publisher's note: Springer Nature remains neutral with regard to jurisdictional claims in published maps and institutional affiliations.

Open Access This article is licensed under a Creative Commons Attribution 4.0 International License, which permits use, sharing, adaptation, distribution and reproduction in any medium or format, as long as you give appropriate credit to the original author(s) and the source, provide a link to the Creative Commons license, and indicate if changes were made. The images or other third party material in this article are included in the article's Creative Commons license, unless indicated otherwise in a credit line to the material. If material is not included in the article's Creative Commons license and your intended use is not permitted by statutory regulation or exceeds the permitted use, you will need to obtain permission directly from the copyright holder. To view a copy of this license, visit http://creativecommons.org/ licenses/by/4.0/.

(C) The Author(s) 2019 